\title{
Superspace first order formalism, trivial symmetries and electromagnetic interactions of linearized supergravity
}

\author{
I.L. Buchbinder, ${ }^{a, b}$ S. James Gates Jr. ${ }^{c, d}$ and K. Koutrolikos ${ }^{c, d}$ \\ ${ }^{a}$ Center of Theoretical Physics, Tomsk State Pedagogical University, \\ 5 Tomsk 634041, Russia \\ ${ }^{b}$ National Research Tomsk State University, \\ 8 Tomsk 634050, Russia \\ ${ }^{c}$ Brown Theoretical Physics Center, \\ Box S, 340 Brook Street, Barus Hall, Providence, RI 02912, U.S.A. \\ ${ }^{d}$ Department of Physics, Brown University, Box 1843, 182 Hope Street, Barus E Holley 545, \\ Providence, RI 02912, U.S.A. \\ E-mail: joseph@tspu.edu.ru, sylvester_gates@brown.edu, \\ konstantinos_koutrolikos@brown.edu
}

AbStRaCT: We introduce a first order description of linearized non-minimal $(n=-1)$ supergravity in superspace, using the unconstrained prepotential superfield instead of the conventionally constrained super one forms. In this description, after integrating out the connection-like auxiliary superfield of first-order formalism, the superspace action is expressed in terms of a single superfield which combines the prepotential and compensator superfields. We use this description to construct the supersymmetric cubic interaction vertex $3 / 2-3 / 2-1 / 2$ which describes the electromagnetic interaction between two nonminimal supergravity multiplets (superspin $Y=3 / 2$ which contains a spin 2 and a spin $3 / 2$ particles) and a vector multiplet (superspin $Y=1 / 2$ contains a spin 1 and a spin $1 / 2$ particles). Exploring the trivial symmetries emerging between the two $Y=3 / 2$ supermultiplets, we show that this cubic vertex must depend on the vector multiplet superfield strength. This result generalize previous results for non-supersymmetric electromagnetic interactions of spin 2 particles. The constructed cubic interaction generates non-trivial deformations of the gauge transformations.

KEYwords: Superspaces, Supersymmetric Effective Theories

ArXiv EPrint: 2107.06854 


\section{Contents}

1 Introduction 1

2 First order formulation $\quad 3$

2.1 Review of first order formulation of free higher spin fields 3

2.2 Superspace first order formulation of vector supermultiplet 4

2.3 Superspace first order formulation of linearized non-minimal supergravity $\quad 7$

3 Trivial symmetries and Noether's procedure $\quad 11$

3.1 Review of trivial symmetries with examples in supersymmetric theories 11

$\begin{array}{ll}3.2 & \text { Application to electromagnetic interactions } \\ \end{array}$

4 Electromagnetic interaction of non-minimal linearized supergravity $\quad 14$

$\begin{array}{lll}4.1 \text { Preparations } & 14\end{array}$

$\begin{array}{ll}4.2 \text { Cubic interaction } 3 / 2-3 / 2-1 / 2 & 16\end{array}$

$\begin{array}{lll}5 & \text { Conclusions } & 17\end{array}$

\section{Introduction}

It has been understood that in the high energy regime $\left(\alpha^{\prime} \rightarrow \infty\right)$ of string theory, the role of higher spin modes is crucial. The unifying framework of the theory, all string excitations must be treated equally, strongly suggests that we should seriously consider higher spin fields and their interactions (preferably with supersymmetry) and overcome the discouragement of early no-go theorems. The investigation of consistent cubic interactions among higher spin fields is a very important first step in this direction. The classification of all non-supersymmetric cubic vertices of massive and massless integer and half-integer higher spin fields was obtained by Metsaev in [1-3] for $d \geq 4$ using light-cone formalism and in $[4,5]$ for $d=3$. More recently a general classification of supersymmetric higher spin multiplets was also found in $[6,7]$.

The covariant construction of such cubic interactions is a highly non-trivial task and most of the successful constructions involve massless fields. In these cases, the gauge redundancy of the higher spin fields is used as a guiding principle which severely constraints the structure of the interaction vertex. A variety of methods have been used to construct such vertices (Noether's procedure [8-14], BRST [15-24], frame-like formulation [25-41]) but all of them are organized in two categories. There is the metric-like description, which uses symmetric, higher rank tensors resembling the metric formulation of gravity and there is the frame-like description which is a generalization of the vielbein description of gravity, where the higher spin fields are described by higher spin algebra valued soldering one forms. 
The metric-like formulation, originates from early works [42-49], offers a more geometric viewpoint which together with the notion of higher spin connections [50] tries to extend our spin 2 intuition to higher spins. This description is very economic in the number of fields it requires. On the other hand the frame formulation, developed by Vasiliev [25, 51], offers a more algebraic approach by extending the notion of Cartan connection in gravity to higher spins and thus resembling the structure of non-abelian Yang-Mills theory for an appropriate underlying higher spin symmetry group. This approach provides an economy of ideas which dictate the dynamics of higher spins and so far it has been more successful in constructing consistent interactions.

For manifestly $N=1$ supersymmetric theories in $4 \mathrm{D}$, the superspace description of free massless higher spins was discovered in [52-55] (see also later discussions in [56-58]) whereas the description of massive higher spin multiplets was only recently discovered in [59] for arbitrary half-integer superspins $(Y=s+1 / 2)$. These formulations use higher rank superfields with both types of spinorial indices symmetrized. They can be understood as the superspace analogs to the geometrical, metric-like approach (see the discussion in [60]) and have been used successfully to construct interactions of higher spin supermultiplets. In [61-68] various non-trivial cubic interactions between higher spin multiplets and matter multiplets have been constructed. Cubic interactions $Y_{1}-Y_{2}-Y_{3}$ among higher spin multiplets with superspins $Y_{1}, Y_{2}, Y_{3}$ were first constructed in $[69,70]$ for the two cases of $Y_{1}=s_{1}+1 / 2$, $Y_{2}=Y_{3}$ (integer or half-integer) and $Y_{1}=s_{1}, Y_{2}=Y_{3}$ (integer or half-integer). These interactions are of the abelian type because the superspace cubic interaction Lagrangian is of the form $\mathcal{L}_{1} \sim \Phi_{1} W_{2} W_{3}$, where $\Phi_{1}$ is the set of superfields that describe the higher spin supermultiplet with superspin $Y_{1}$ and $W_{2}, W_{3}$ are the gauge invariant superfield strengths for higher spin multiplets with superspins $Y_{2}, Y_{3}$ respectively. Because of their structure these interactions do not generate corrections to the free gauge transformations.

To make progress in the program of constructing manifestly supersymmetric cubic interactions of higher spins, we would like to investigate interactions of the type $\mathcal{L}_{1} \sim$ $\Phi_{1} \Phi_{2} W_{3}$. Such cubic interactions have been recently been constructed for higher spin theories with on-shell supersymmetry [71]. In general, this type of interactions generate non-trivial deformations of the gauge transformations that may also deform the gauge algebra. An important subclass of such interactions are the electromagnetic interactions of higher spin multiplets, $\mathrm{Y}-\mathrm{Y}-1 / 2$. By turning on the electric charge of a superspin $\mathrm{Y}$ we can couple it with the vector multiplet $(Y=1 / 2)$. For electromagnetic interactions one must consider a doublet of (super)fields in order for the $\mathrm{U}(1)$ group (isomorphic to $\mathrm{SO}(2)$ ) which controls the interaction to be able to be realized. ${ }^{1}$ Such interactions have been explicitly constructed for non-supersymmetric theories in [72-75] and motivate us to find the corresponding manifestly supersymmetric structures.

In this paper we focus on the first member of this class of interactions, the electromagnetic interactions of linearized supergravity $(\mathrm{Y}=3 / 2): 3 / 2-3 / 2-1 / 2 .^{2}$ Specifically,

\footnotetext{
${ }^{1}$ One can also understand the emergence of the doublet as a consequence of charge conservation on the cubic vertex, meaning that if we turn on the electric charge for one higher spin multiplet we must also have another one with opposite charge.

${ }^{2}$ Not to be confused with the (super)gravitational interaction of the vector multiplet: $3 / 2-1 / 2-1 / 2$.
} 
we consider a doublet of linearized non-minimal $(n=-1)$ supergravity supermultiplets described by superfields $H_{\alpha \dot{\alpha}}^{i}, \chi_{\alpha}^{i}, i=1,2$ and a vector multiplet described by superfield $V$. By exploiting the set of trivial symmetries of free theory, we show that the cubic interaction must depend purely on the superfield strength of the vector multiplet $W_{\alpha}$ and does not include bare $V$ terms, thus making it trivially invariant under the vector gauge transformation. On the other hand, the linearized supergravity superfields will participate bare and via Noether's procedure a deformation of the free gauge transformations will be required. We are using non-minimal supergravity because, unlike the minimal formulations, it is a geometrical theory ${ }^{3}$ and it is the lowest member of a tower of non-minimal higher spin supermultiplets, as observed in [53]. Therefore any intuition we gain by studying non-minimal supergravity will benefit us towards constructing cubic interactions of supersymmetric higher spin multiplets.

In order to construct this interaction (and being motivated by the success of the framelike formulation) we develop a superspace, first order formulation of linearized supergravity by introducing a not fully symmetric superfield $\mathcal{I}_{\beta \alpha \dot{\alpha}}$ which is invariant under a relaxed set of gauge transformations and combines superfields $H_{\alpha \dot{\alpha}}$ and $\chi_{\alpha}$. We show that the linearized non-minimal supergravity action can be written only in terms of $\mathcal{I}_{\beta \alpha \dot{\alpha}}$ and is the appropriate superfield variable to construct the cubic interaction with the vector multiplet.

The paper is organized as follows. In section 2, we introduce the first order formulation of linearized non-minimal supergravity in terms of the superfield $\mathcal{I}_{\beta \alpha \dot{\alpha}}$ and a pair of auxiliary connection-like superfields. In section 3 , we review the notion of trivial symmetries and give examples of their application in supersymmetric theories. In this case the doublet of superfields gives rise to such trivial symmetries which in turn constraint the cubic interaction to depend on the superfield strength $W_{\alpha}$ of the vector multiplet instead of having bare superfield V terms. Finally, in section 4 using Noether's procedure we construct the superspace cubic interaction Lagrangian and find the coupling constant corrections to the free gauge transformations.

\section{First order formulation}

\subsection{Review of first order formulation of free higher spin fields}

The power of first order formalism has been demonstrated repeatedly in the case of gravity, supergravity and higher spins. Some of its characteristic features are (i) the independence between the vielbein and the spin connection, (ii) the algebraic equation of motion for the spin connection, which can be solved in terms of the vielbein field (and matter or gauge fields when present) and its derivative (iii) the (algebraic) local Lorentz symmetry of the vielbein that reduces its degrees of freedom to match that of the metric.

For higher spin fields a frame-like formalism has been developed by Vasiliev [25, 51]. The key observation is that starting from the metric-like formulation using fully symmetric tensors $h_{m_{1} m_{2} \ldots m_{s}}(\overbrace{\square \square}^{s})$ with appropriate gauge transformations $\delta h_{m_{1} m_{2} \ldots m_{s}}=$

\footnotetext{
${ }^{3}$ In the sense that its equations of motion can be written purely in terms of de Wit-Freedman-like superconnections as found in [60].
} 
$\partial_{\left(m_{1}\right.} \xi_{\left.m_{2} \ldots m_{s}\right)}$ one can relax the symmetry for one of the indices and define a not fully symmetric tensor field $e_{n m_{1} \ldots m_{s-1}}(\square \otimes \overbrace{\square \square \square}^{s-1})$ with gauge transformation $\delta e_{n m_{1} \ldots m_{s-1}}=$ $\partial_{n} \xi_{m_{1} \ldots m_{s-1}}$. The relaxed gauge transformation allows the construction of a first order, gauge invariant quantity

$$
I_{m_{1} \ldots m_{s-1}}^{k l} \sim \epsilon^{p n k l} \partial_{p} e_{n m_{1} \ldots m_{s-1}}
$$

which can be used to write a trivially invariant action $S[I]$. However, in order to maintain the same number of degrees of freedom one must introduce an additional algebraic local symmetry $\delta e_{n m_{1} \ldots m_{s-1}}=a_{n m_{1} \ldots m_{s-1}}$, where $a_{n m_{1} \ldots m_{s-1}}$ is an $(s-1,1)$ tableau

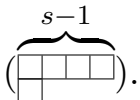
Checking the invariance of $S[I]$ with respect to this local symmetry becomes more involved. This process is simplified by realizing that $I^{k l}{ }_{m_{1} \ldots m_{s-1}}$ satisfies the following identity

$$
\partial_{k} I^{k l}{ }_{m_{1} \ldots m_{s-1}}=0
$$

which can be promoted to a Bianchi identity that enforces the $a$-transformation. This is achieved by introducing an auxiliary field, the connection, $\omega_{r n m_{1} \ldots m_{s-1}}(\square \otimes \overbrace{\square_{\square}^{s-1}}^{\text {Th }})$ with a transformation law $\delta \omega_{r n m_{1} \ldots m_{s-1}}=\partial_{r} a_{n m_{1} \ldots m_{s-1}}$. In this description the action takes symbolically the form

$$
S[\omega, I] \sim \int \omega^{2}+\omega I .
$$

This action provides a first order formulation of the usual Fronsdal description $[48,49]$ and by integrating out the connection ${ }^{4}$ one recovers the action $S[I]$.

\subsection{Superspace first order formulation of vector supermultiplet}

It would be useful to have a similar first order description in superspace where supersymmetry is manifest. Of course, there is the example of supergravity in superspace which utilizes supervielbeins and superconnections [76-78] (for details see [79-81]). However such objects carry many more degrees of freedom and do not describe irreducible representations of the super-Poincare group. Various sets of constraints have to be imposed which are solved in terms of prepotential superfields. The linearization of this description for the case of non-minimal supergravity will give (2.20). By doing all that (solving the constraints and linearizing the theory), the notion of a first order formulation, as described above, has faded and we effectively converted back to a metric-like description. Therefore, our aspiration to acquire a first order formulation of linearized supergravity and other higher spin supermultiplets that can be used to construct cubic interactions is a well motivated one. In order to find one, we follow the spirit of the discussion of section 2.1 but we use the unconstrained prepotential superfields instead of the conventional superframes.

\footnotetext{
${ }^{4}$ For $s>2$ the action has an additional symmetry $\delta \omega_{r n m_{1} \ldots m_{s-1}}=\sigma_{r n m_{1} \ldots m_{s-1}}$, where $\sigma_{r n m_{1} \ldots m_{s-1}}$

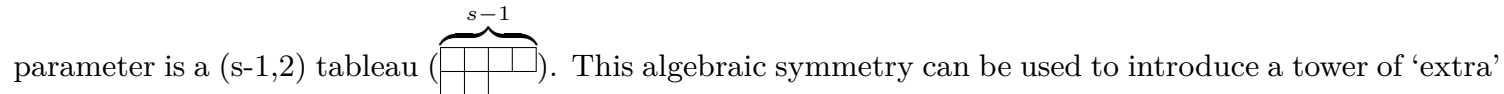
fields [51] which decouple at the free theory limit in order to get second order equations of motion and can be understood as a realization of the de Wit-Freedman connections.
} 
It will be instructive to start our consideration with a simpler supersymmetric multiplet, the vector multiplet $(Y=1 / 2)$. The superspace description of the vector multiplet is given by a real, scalar superfield $V$. Its dynamics are determined by the following superspace action

$$
S_{(\mathrm{Y}=1 / 2)}[V]=\int d^{8} z V \mathrm{D}^{\gamma} \overline{\mathrm{D}}^{2} \mathrm{D}_{\gamma} V
$$

which is invariant under the gauge transformation

$$
\delta V=\mathrm{D}^{2} \bar{L}+\overline{\mathrm{D}}^{2} L .
$$

As discussed in 2.1 we have to relax a property of the superfield $V$ which in turn will simplify the gauge transformation and help us define a lower order invariant quantity. In the case of spin 2 that property was the symmetrization of the two spacetime indices. In this case, the superfield $V$ does not carry any indices, so there is no symmetrization to relax. The only property that $V$ carries that can be modified is it's reality $(V=\bar{V})$. Therefore, we drop the reality condition of $V$ and instead we introduce a complex scalar superfield $\mathcal{V}$ with the gauge transformation

$$
\delta_{L} \mathcal{V}=\mathrm{D}^{2} \bar{L}
$$

Due to the algebra of the supersymmetric covariant derivatives, one can immediately write a trivially gauge invariant quantity

$$
\mathcal{I}_{\alpha}=\mathrm{D}_{\alpha} \mathcal{V}, \delta_{L} \mathcal{I}_{\alpha}=0
$$

By complexifying $V$ we introduced extra degrees of freedom which must be removed. Following the philosophy of first order formulation, we introduce an additional algebraic local symmetry

$$
\delta_{\eta} \mathcal{V}=i \eta, \eta=\bar{\eta}
$$

which removes the imaginary part of $\mathcal{V}$ and thus restoring the degrees of freedom. Under this additional $\eta$-transformation $\mathcal{I}_{\alpha}$ changes as follows:

$$
\delta_{\eta} \mathcal{I}_{\alpha}=i \mathrm{D}_{\alpha} \eta
$$

The action will be constructed in terms of $\mathcal{I}_{\alpha}$, making it trivially invariant under $L$ - transformations. For the $\eta$-invariance we introduce an auxiliary superfield $\Omega_{\alpha}$ playing the role of the connection and we select $\Omega_{\alpha}$ 's $\eta$-transformations such that the action is also $\eta$-invariant. Because of it's auxiliary nature, $\Omega_{\alpha}$ must appear in the action algebraically, hence one is tempted to write an action similar to (2.3). However, due to the engineering dimensions of the superspace integration measure this is impossible. The solution is that one is forced to introduce two connection-like superfields $\mathcal{W}_{\alpha}$ and $\Omega_{\alpha}$. This is a familiar characteristic feature of supersymmetric theories were the fermionic auxiliary (super)fields must appear in pairs [82] because they carry half odd-integer engineering dimensions and the superspace 
Lagrangian has dimension 2 in four dimensions. Now, with all that in mind, we can write the most general ansatz for the action:

$$
S[\mathcal{W}, \Omega, \mathcal{I}]=\int d^{8} z\left[c \mathcal{W}^{\alpha} \Omega_{\alpha}+\mathcal{W}^{\alpha} \mathcal{I}_{\alpha}+\Omega^{\alpha}\left\{f_{1} \overline{\mathrm{D}}^{2} \mathcal{I}_{\alpha}+f_{2} \mathrm{D}_{\alpha} \overline{\mathrm{D}}^{\dot{\alpha}} \overline{\mathcal{I}}_{\dot{\alpha}}+f_{3} \overline{\mathrm{D}}^{\dot{\alpha}} \mathrm{D}_{\alpha} \overline{\mathcal{I}}_{\dot{\alpha}}\right\}+c . c .\right]
$$

where coefficients $f_{1}, f_{2}, f_{3}$ are to be determined. ${ }^{5}$ The variation of this action can be organized as follows:

$$
\begin{aligned}
\delta S[\mathcal{W}, \Omega, \mathcal{I}]=\int d^{8} z[ & \delta \mathcal{W}^{\alpha} \mathcal{I}_{\alpha}+\delta \Omega^{\alpha}\left\{f_{1} \overline{\mathrm{D}}^{2} \mathcal{I}_{\alpha}+f_{2} \mathrm{D}_{\alpha} \overline{\mathrm{D}}^{\dot{\alpha}} \overline{\mathcal{I}}_{\dot{\alpha}}+f_{3} \overline{\mathrm{D}}^{\dot{\alpha}} \mathrm{D}_{\alpha} \overline{\mathcal{I}}_{\dot{\alpha}}\right\}+c . c . \\
& +\mathcal{W}^{\alpha}\left\{c \delta \Omega_{\alpha}+\delta \mathcal{I}_{\alpha}\right\}+c . c . \\
& \left.+\Omega^{\alpha}\left\{c \delta \mathcal{W}_{\alpha}+f_{1} \overline{\mathrm{D}}^{2} \delta \mathcal{I}_{\alpha}+f_{2} \mathrm{D}_{\alpha} \overline{\mathrm{D}}^{\dot{\alpha}} \delta \overline{\mathcal{I}}_{\dot{\alpha}}+f_{3} \overline{\mathrm{D}}^{\dot{\alpha}} \mathrm{D}_{\alpha} \delta \overline{\mathcal{I}}_{\dot{\alpha}}\right\}+c . c .\right]
\end{aligned}
$$

Both $\eta$ and $L$-transformations of $\Omega_{\alpha}$ and $\mathcal{W}_{\alpha}$ are chosen such that the last two lines in (2.11) vanish

$$
\begin{array}{ll}
\delta \Omega_{\alpha}=-\frac{1}{c} \delta \mathcal{I}_{\alpha}, & \delta \mathcal{W}_{\alpha}=-\frac{1}{c}\left\{f_{1} \overline{\mathrm{D}}^{2} \delta \mathcal{I}_{\alpha}+f_{2} \mathrm{D}_{\alpha} \overline{\mathrm{D}}^{\dot{\alpha}} \delta \overline{\mathcal{I}}_{\dot{\alpha}}+f_{3} \overline{\mathrm{D}}^{\dot{\alpha}} \mathrm{D}_{\alpha} \delta \overline{\mathcal{I}}_{\dot{\alpha}}\right\} \\
\delta_{L} \Omega_{\alpha}=0, & \delta_{\eta} \Omega_{\alpha}=-\frac{i}{c} \mathrm{D}_{\alpha} \eta, \\
\delta_{L} \mathcal{W}_{\alpha}=0, & \delta_{\eta} \mathcal{W}_{\alpha}=-\frac{i}{c}\left(f_{1}+f_{3}\right) \overline{\mathrm{D}}^{2} \mathrm{D}_{\alpha} \eta+\frac{i}{c}\left(2 f_{2}-f_{3}\right) \mathrm{D}_{\alpha} \overline{\mathrm{D}}^{2} \eta
\end{array}
$$

Substituting (2.12a) back in (2.11) gives

$$
0=\delta_{\eta} S[\mathcal{W}, \Omega, \mathcal{I}]=\frac{2 i}{c} \int d^{8} z \eta\left\{\left(f_{1}+f_{3}\right) \mathrm{D}^{\alpha} \overline{\mathrm{D}}^{2} \mathcal{I}_{\alpha}+\left(f_{3}-2 f_{2}\right) \overline{\mathrm{D}}^{2} \mathrm{D}^{\alpha} \mathcal{I}_{\alpha}\right\}+c . c .
$$

and fixes the coefficients

$$
f_{1}=-2 f_{2}, f_{3}=2 f_{2}
$$

With this choice, the action becomes:

$S[\mathcal{W}, \Omega, \mathcal{I}]=\int d^{8} z\left[c \mathcal{W}^{\alpha} \Omega_{\alpha}+\mathcal{W}^{\alpha} \mathcal{I}_{\alpha}+f_{2} \Omega^{\alpha}\left\{-2 \overline{\mathrm{D}}^{2} \mathcal{I}_{\alpha}+\mathrm{D}_{\alpha} \overline{\mathrm{D}}^{\dot{\alpha}} \overline{\mathcal{I}}_{\dot{\alpha}}+2 \overline{\mathrm{D}}^{\dot{\alpha}} \mathrm{D}_{\alpha} \overline{\mathcal{I}}_{\dot{\alpha}}\right\}+\right.$ c.c. $]$

The equations of motion of $\mathcal{W}_{\alpha}$ and $\Omega_{\alpha}$ are algebraic

$$
c \Omega_{\alpha}+\mathcal{I}_{\alpha}=0, c \mathcal{W}_{\alpha}+f_{2}\left\{-2 \overline{\mathrm{D}}^{2} \mathcal{I}_{\alpha}+\mathrm{D}_{\alpha} \overline{\mathrm{D}}^{\dot{\alpha}} \overline{\mathcal{I}}_{\dot{\alpha}}+2 \overline{\mathrm{D}}^{\dot{\alpha}} \mathrm{D}_{\alpha} \overline{\mathcal{I}}_{\dot{\alpha}}\right\}=0
$$

and thus we can integrate them out. The result is the following action

$$
S_{(\mathrm{Y}=1 / 2)}\left[\mathcal{I}_{\alpha}\right]=-\frac{2 f_{2}}{c} \int d^{8} z\left\{\mathcal{I}^{\alpha} \mathrm{D}_{\alpha} \overline{\mathrm{D}}^{\dot{\alpha}} \overline{\mathcal{I}}_{\dot{\alpha}}+2 \mathcal{I}^{\alpha} \overline{\mathrm{D}}^{\dot{\alpha}} \mathrm{D}_{\alpha} \overline{\mathcal{I}}_{\dot{\alpha}}-\left(\mathcal{I}^{\alpha} \overline{\mathrm{D}}^{2} \mathcal{I}_{\alpha}+\text { c.c. }\right)\right\} .
$$

\footnotetext{
${ }^{5}$ Notice that the term $\mathrm{D}^{2} \mathcal{I}_{\alpha}$ is not present inside the curly bracket. This term is zero due to (2.7).
} 
Using the definition of $\mathcal{I}_{\alpha}$ one can see that this action is equal to $S_{(\mathrm{Y}=1 / 2)}[V]$. The equation of motion for superfield $\mathcal{V}$ in this language takes the form

$$
\mathcal{E}^{(\mathcal{V})}=\mathrm{D}^{\alpha} \overline{\mathrm{D}}^{2} \mathcal{I}_{\alpha}+\overline{\mathrm{D}}^{\dot{\alpha}} \mathrm{D}^{2} \overline{\mathcal{I}}_{\dot{\alpha}}=\mathrm{D}^{\gamma} \overline{\mathrm{D}}^{2} \mathrm{D}_{\gamma}(\mathcal{V}+\overline{\mathcal{V}})=\mathrm{D}^{\alpha} W_{\alpha}
$$

where $W_{\alpha}=\overline{\mathrm{D}}^{2} \mathrm{D}_{\alpha} V$ is the gauge invariant superfield strength for the vector multiplet and $V=\mathcal{V}+\overline{\mathcal{V}}$. This equation of motion satisfies the following Bianchi identity

$$
\mathcal{E}^{(\mathcal{V})}=\overline{\mathcal{E}}^{(\mathcal{V})}
$$

which is responsible for the $\eta$-invariance of $S_{(\mathrm{Y}=1 / 2)}\left[\mathcal{I}_{\alpha}\right]$.

\subsection{Superspace first order formulation of linearized non-minimal supergravity}

The superspace description of the non-minimal superspin $Y=3 / 2$ supermultiplet is given in terms of an unconstrained, real, gauge superfield $H_{\alpha \dot{\alpha}}$ and an unconstrained fermionic compensator superfield $\chi_{\alpha}$ (we follow $[57,83]$ ). The action is

$$
\begin{aligned}
S_{(\mathrm{Y}=3 / 2)}\left[H_{\alpha \dot{\alpha}}, \chi_{\alpha}\right]=\int d^{8} z\{ & H^{\alpha \dot{\alpha}} \mathrm{D}^{\gamma} \overline{\mathrm{D}}^{2} \mathrm{D}_{\gamma} H_{\alpha \dot{\alpha}}+2 \chi^{\alpha} \mathrm{D}_{\alpha} \overline{\mathrm{D}}^{\dot{\alpha}} \bar{\chi}_{\dot{\alpha}} \\
& \left.-2 H^{\alpha \dot{\alpha}} \overline{\mathrm{D}}_{\dot{\alpha}} \mathrm{D}^{2} \chi_{\alpha}-2 \chi^{\alpha} \mathrm{D}^{2} \chi_{\alpha}+\text { c.c. }\right\}
\end{aligned}
$$

and is invariant under the following transformations

$$
\delta H_{\alpha \dot{\alpha}}=\mathrm{D}_{\alpha} \bar{L}_{\dot{\alpha}}-\overline{\mathrm{D}}_{\dot{\alpha}} L_{\alpha}, \delta \chi_{\alpha}=\overline{\mathrm{D}}^{2} L_{\alpha}+\mathrm{D}^{\beta} \Lambda_{\alpha \beta}, \Lambda_{\alpha \beta}=\Lambda_{\beta \alpha} .
$$

The gauge invariant superfield strength is

$$
W_{\alpha \beta \gamma} \sim \overline{\mathrm{D}}^{2} \mathrm{D}_{(\alpha} \partial_{\beta}{ }^{\dot{\gamma}} H_{\gamma) \dot{\gamma}} .
$$

Similar to the discussion in 2.2 , superfield $H_{\alpha \dot{\alpha}}$ has no symmetrization between indices, hence the only possible modification that one can do is to relax its reality condition. Therefore, we introduce a complex superfield $\mathcal{H}_{\alpha \dot{\alpha}}$ with the same index structure as the original $H$-superfield, equipped with the gauge transformation

$$
\delta_{L} \mathcal{H}_{\alpha \dot{\alpha}}=\mathrm{D}_{\alpha} \bar{L}_{\dot{\alpha}}
$$

and an additional algebraic local symmetry that will fix the increase in the degrees of freedom

$$
\delta_{\eta} \mathcal{H}_{\alpha \dot{\alpha}}=i \eta_{\alpha \dot{\alpha}}, \eta_{\alpha \dot{\alpha}}=\bar{\eta}_{\alpha \dot{\alpha}}
$$

Next, we use the $L$-transformation (2.23) to find an invariant quantity that can be used to make the action manifestly invariant. If we try something similar to $(2.7)$ we get $\mathcal{I}_{\beta \alpha \dot{\alpha}}=$ $\mathrm{D}_{\beta} \mathcal{H}_{\alpha \dot{\alpha}}$, but it is obvious that this is no longer an $L$-invariant because $\delta \mathcal{I}_{\beta \alpha \dot{\alpha}}=-C_{\beta \alpha} \mathrm{D}^{2} \bar{L}_{\dot{\alpha}}$, where $C_{\beta \alpha}$ is the antisymmetric spinorial metric. ${ }^{6}$ This can be fixed by introducing a compensating superfield with an appropriate $L$-transformation. We introduce a fermionic compensator $\mathcal{X}_{\alpha}$ with the transformation

$$
\delta_{L} \mathcal{X}_{\alpha}=\overline{\mathrm{D}}^{2} L_{\alpha}, \delta_{\eta} \mathcal{X}_{\alpha}=0
$$

\footnotetext{
${ }^{6}$ We use Superspace [79] conventions.
} 
The introduction of $\mathcal{X}_{\alpha}$ allows us to combine it with $\mathcal{H}_{\alpha \dot{\alpha}}$ and define the following $L$ invariant quantity

$$
\mathcal{I}_{\beta \alpha \dot{\alpha}}=\mathrm{D}_{\beta} \mathcal{H}_{\alpha \dot{\alpha}}+C_{\beta \alpha} \overline{\mathcal{X}}_{\dot{\alpha}}, \delta_{L} \mathcal{I}_{\beta \alpha \dot{\alpha}}=0
$$

The difference between (2.7) and (2.26) is qualitative and can be traced back to the different number of supersymmetric covariant derivatives participate in their corresponding $L$-transformations (2.6) and (2.23). The $\eta$-transformation of $\mathcal{I}_{\beta \alpha \dot{\alpha}}$ can be easily found to be

$$
\delta_{\eta} \mathcal{I}_{\beta \alpha \dot{\alpha}}=i \mathrm{D}_{\beta} \eta_{\alpha \dot{\alpha}}
$$

The action will be written in terms of $\mathcal{I}_{\beta \alpha \dot{\alpha}}$ and it's derivatives, making it manifestly $L$-invariant. For the $\eta$-symmetry we rely on the critical role of the auxiliary connectionlike superfields we have to introduce. As discussed in 2.2, because these superfields are fermionic in nature, must come in pairs $\left(\mathcal{W}_{\beta \alpha \dot{\alpha}}, \Omega_{\beta \alpha \dot{\alpha}}\right)$. The general ansatz for this action (up to redefinitions) takes the form

$$
S[\mathcal{W}, \Omega, \mathcal{I}]=\int d^{8} z\left\{\mathcal{W}^{\beta \alpha \dot{\alpha}} \Omega_{\beta \alpha \dot{\alpha}}+\mathcal{W}^{\beta \alpha \dot{\alpha}} \mathcal{I}_{\beta \alpha \dot{\alpha}}+\Omega^{\beta \alpha \dot{\alpha}} \mathcal{J}_{\beta \alpha \dot{\alpha}}\right\}+c . c .
$$

where

$$
\begin{aligned}
\mathcal{J}_{\beta \alpha \dot{\alpha}}= & f_{1} \mathrm{D}^{2} \mathcal{I}_{\beta \alpha \dot{\alpha}}+f_{2} \overline{\mathrm{D}}^{2} \mathcal{I}_{\beta \alpha \dot{\alpha}}+f_{3} \mathrm{D}_{\beta} \overline{\mathrm{D}}^{\dot{\beta}} \overline{\mathcal{I}}_{\alpha \dot{\beta} \dot{\alpha}}+f_{4} \overline{\mathrm{D}}^{\dot{\beta}} \mathrm{D}_{\beta} \overline{\mathcal{I}}_{\alpha \dot{\beta} \dot{\alpha}} \\
& +g_{1} C_{\beta \alpha} \overline{\mathrm{D}}^{2} \mathcal{I}_{\dot{\alpha}}+g_{2} \mathrm{D}_{\beta} \overline{\mathrm{D}}_{\dot{\alpha}} \overline{\mathcal{I}}_{\alpha}+g_{3} \overline{\mathrm{D}}_{\dot{\alpha}} \mathrm{D}_{\beta} \overline{\mathcal{I}}_{\alpha} \\
& +d_{1} C_{\beta \alpha} \mathrm{D}^{\gamma} \overline{\mathrm{D}}_{\dot{\alpha}} \overline{\mathcal{I}}_{\gamma}+d_{2} C_{\beta \alpha} \overline{\mathrm{D}}_{\dot{\alpha}} \mathrm{D}^{\gamma} \overline{\mathcal{I}}_{\gamma}
\end{aligned}
$$

and $\mathcal{I}_{\dot{\alpha}}=C^{\beta \alpha} \mathcal{I}_{\beta \alpha \dot{\alpha}}$. The variation of this action will generate six terms that can be organized in the following way

$$
\begin{aligned}
\delta S[\mathcal{W}, \Omega, \mathcal{I}]=\int d^{8} z\{ & \delta \mathcal{W}^{\beta \alpha \dot{\alpha}} \mathcal{I}_{\beta \alpha \dot{\alpha}}+\delta \Omega^{\beta \alpha \dot{\alpha}} \mathcal{J}_{\beta \alpha \dot{\alpha}}+\text { c.c. } \\
& +\mathcal{W}^{\beta \alpha \dot{\alpha}}\left\{\delta \Omega_{\beta \alpha \dot{\alpha}}+\delta \mathcal{I}_{\beta \alpha \dot{\alpha}}\right\}+\text { c.c. } \\
& \left.+\Omega^{\beta \alpha \dot{\alpha}}\left\{\delta \mathcal{W}_{\beta \alpha \dot{\alpha}}+\delta \mathcal{J}_{\beta \alpha \dot{\alpha}}\right\}+\text { c.c. }\right\} .
\end{aligned}
$$

This is suggestive of the transformation laws that we should assign to $\mathcal{W}_{\beta \alpha \dot{\alpha}}$ and $\Omega_{\beta \alpha \dot{\alpha}}$ :

$$
\begin{array}{ll}
\delta \Omega_{\beta \alpha \dot{\alpha}}=-\delta \mathcal{I}_{\beta \alpha \dot{\alpha}}, \quad \delta \mathcal{W}_{\beta \alpha \dot{\alpha}}= & -\delta \mathcal{J}_{\beta \alpha \dot{\alpha}}, \\
\delta_{L} \Omega_{\beta \alpha \dot{\alpha}}=0, \quad \delta_{\eta} \Omega_{\beta \alpha \dot{\alpha}}= & -i \mathrm{D}_{\beta} \eta_{\alpha \dot{\alpha}}, \\
\delta_{L} \mathcal{W}_{\beta \alpha \dot{\alpha}}=0, \quad \delta_{\eta} \mathcal{W}_{\beta \alpha \dot{\alpha}}= & -i\left(f_{2}+f_{4}\right) \overline{\mathrm{D}}^{2} \mathrm{D}_{\beta} \eta_{\alpha \dot{\alpha}}+i\left(2 f_{3}-f_{4}-g_{2}\right) \mathrm{D}_{\beta} \overline{\mathrm{D}}^{2} \eta_{\alpha \dot{\alpha}} \\
& +i g_{1} C_{\beta \alpha} \overline{\mathrm{D}}^{2} \mathrm{D}^{\gamma} \eta_{\gamma \dot{\alpha}}-i d_{1} C_{\beta \alpha} \mathrm{D}^{\gamma} \overline{\mathrm{D}}^{2} \eta_{\gamma \dot{\alpha}} \\
& +i g_{3} \overline{\mathrm{D}}_{\dot{\alpha}} \mathrm{D}_{\beta} \overline{\mathrm{D}}^{\dot{\gamma}} \eta_{\alpha \dot{\gamma}}+i d_{2} C_{\beta \alpha} \overline{\mathrm{D}}_{\dot{\alpha}} \mathrm{D}^{\gamma} \overline{\mathrm{D}}^{\dot{\gamma}} \eta_{\gamma \dot{\gamma}} .
\end{array}
$$


Substituting the above transformations to (2.30) we find that the $\eta$-variation of the action is

$$
\begin{aligned}
\delta_{\eta} S[\mathcal{W}, \Omega, \mathcal{I}]=\int d^{8} z i \eta^{\alpha \dot{\alpha}}\{ & 2\left(f_{2}+f_{4}\right) \mathrm{D}^{\beta} \overline{\mathrm{D}}^{2} \mathcal{I}_{\beta \alpha \dot{\alpha}}-\left(4 f_{3}-2 f_{4}-g_{2}\right) \overline{\mathrm{D}}^{2} \mathrm{D}^{\beta} \mathcal{I}_{\beta \alpha \dot{\alpha}} \\
& -g_{3} \overline{\mathrm{D}}_{\dot{\alpha}} \mathrm{D}^{\beta} \overline{\mathrm{D}}^{\dot{\beta}} \mathcal{I}_{\beta \alpha \dot{\beta}}+\left(2 g_{1}-g_{3}\right) \mathrm{D}_{\alpha} \overline{\mathrm{D}}^{2} \mathcal{I}_{\dot{\alpha}} \\
& \left.+\left(-2 d_{1}+2 g_{2}-g_{3}\right) \overline{\mathrm{D}}^{2} \mathrm{D}_{\alpha} \mathcal{I}_{\dot{\alpha}}+2 d_{2} \overline{\mathrm{D}}_{\dot{\alpha}} \mathrm{D}_{\alpha} \overline{\mathrm{D}}^{\dot{\beta}} \mathcal{I}_{\dot{\beta}}\right\}+ \text { c.c. }
\end{aligned}
$$

Because the terms inside the bracket are independent (not related algebraically), the $\eta$ invariance of the action is achieved by choosing

$$
f_{2}=-f_{4}, g_{2}=4 f_{3}-2 f_{4}, g_{3}=0, g_{1}=0, d_{1}=g_{2}, d_{2}=0 .
$$

Unlike the vector multiplet case, this procedure did not fix all the parameters and there is still plenty of freedom left parametrized by coefficients $f_{1}, f_{3}, f_{4}$. This is a signal that there is some further structure to be explored. Interestingly, one can check that $\mathcal{I}_{\beta \alpha \dot{\alpha}}$ satisfies the following two identities:

$$
\begin{aligned}
& \mathrm{D}_{(\gamma} \mathcal{I}_{\beta \alpha) \dot{\alpha}}=0, \\
& \mathrm{D}_{(\beta} \overline{\mathrm{D}}^{\dot{\alpha}} \mathrm{D}_{\alpha)} \mathcal{I}_{\dot{\alpha}}-2 \mathrm{D}_{(\beta} \overline{\mathrm{D}}^{\dot{\alpha}} \mathrm{D}^{\gamma} \mathcal{I}_{|\gamma| \alpha) \dot{\alpha}}+3 \mathrm{D}^{2} \overline{\mathrm{D}}^{\dot{\alpha}} \mathcal{I}_{(\beta \alpha) \dot{\alpha}}=0 .
\end{aligned}
$$

Interpreting these two identities as hints for additional symmetries, we promote them to Bianchi identities which are responsible for the invariance of the action under these symmetries. For example, (2.36a) suggests that in the $\mathcal{W}^{\beta \alpha \dot{\alpha}} \mathcal{I}_{\beta \alpha \dot{\alpha}}$ term of the action (2.28) we can shift $\mathcal{W}_{\beta \alpha \dot{\alpha}} \rightarrow \mathcal{W}_{\beta \alpha \dot{\alpha}}+\mathrm{D}^{\gamma} \Lambda_{\gamma \beta \alpha \dot{\alpha}}$ for some $\Lambda_{\gamma \beta \alpha \dot{\alpha}}$ which is symmetric in the undotted indices. To explore this possibility we introduce a new transformation

$$
\delta_{\Lambda} \mathcal{H}=0, \delta_{\Lambda} \mathcal{X}_{\alpha}=\mathrm{D}^{\gamma} \Lambda_{\gamma \alpha}, \Lambda_{\alpha \beta}=\Lambda_{\beta \alpha} \Rightarrow \delta_{\Lambda} \mathcal{I}_{\beta \alpha \dot{\alpha}}=-C_{\beta \alpha} \overline{\mathrm{D}}^{\dot{\gamma}} \bar{\Lambda}_{\dot{\gamma} \dot{\alpha}}
$$

Using rule (2.31) we find the appropriate $\Lambda$-transformations for the two connection-like superfields

$$
\begin{aligned}
\delta_{\Lambda} \Omega_{\beta \alpha \dot{\alpha}}=C_{\beta \alpha} \overline{\mathrm{D}}^{\dot{\gamma}} \bar{\Lambda}_{\dot{\gamma} \dot{\alpha}}, \delta_{\Lambda} \mathcal{W}_{\beta \alpha \dot{\alpha}}= & f_{1} C_{\beta \alpha} \mathrm{D}^{2} \overline{\mathrm{D}}^{\dot{\gamma}} \Lambda_{\dot{\gamma} \dot{\alpha}}+\left(f_{3}-2 g_{2}\right) \mathrm{D}_{\beta} \overline{\mathrm{D}}_{\dot{\alpha}} \mathrm{D}^{\gamma} \Lambda_{\gamma \alpha} \\
& +\left(2 g_{3}-f_{4}\right) \overline{\mathrm{D}}_{\dot{\alpha}} \mathrm{D}^{2} \Lambda_{\beta \alpha}-2 d_{1} C_{\beta \alpha} \mathrm{D}^{\gamma} \overline{\mathrm{D}}_{\dot{\alpha}} \mathrm{D}^{\rho} \Lambda_{\rho \gamma}
\end{aligned}
$$

and the $\Lambda$-variation of the action (using (2.30) and (2.36b)) becomes

$$
\begin{array}{r}
\delta_{\Lambda} S[\mathcal{W}, \Omega, \mathcal{I}]=\int d^{8} z \Lambda^{\gamma \alpha}\left\{-2 f_{1} \mathrm{D}_{\gamma} \overline{\mathrm{D}}^{2} \overline{\mathcal{I}}_{\alpha}-2\left(f_{3}-2 g_{2}+4 d_{1}\right) \mathrm{D}_{\gamma} \overline{\mathrm{D}}^{2} \mathrm{D}^{\beta} \mathcal{I}_{\beta \alpha \dot{\alpha}}\right. \\
\left.+\left(2 f_{4}-2 g_{3}+12 d_{1}-3 g_{2}\right) \mathrm{D}^{2} \overline{\mathrm{D}}^{\dot{\alpha}} \mathcal{I}_{\gamma \alpha \dot{\alpha}}\right\}+c . c .
\end{array}
$$

The $\Lambda$-invariance of the action fixes the remaining free parameters such that

$$
f_{1}=0, f_{3}=2 g_{2}-4 d_{1}, f_{4}=g_{3}-6 d_{1}+\frac{3}{2} g_{2}
$$


which together with (2.35) fix all coefficients:

$$
f_{1}=0, f_{2}=-f_{4}, f_{3}=\frac{4}{9} f_{4}, g_{1}=0, g_{2}=-\frac{2}{9} f_{4}, g_{3}=0, \quad d_{1}=-\frac{2}{9} f_{4}, d_{2}=0 \text {. }
$$

For these coefficients the $\Lambda$-transformation of $\mathcal{W}_{\beta \alpha \dot{\alpha}}$ takes the form

$$
\delta_{\Lambda} \mathcal{W}_{\beta \alpha \dot{\alpha}}=\frac{4}{33 !} \mathrm{D}^{\gamma} \overline{\mathrm{D}}_{\dot{\alpha}} \mathrm{D}_{(\gamma} \Lambda_{\beta \alpha)}+\frac{4}{3} \mathrm{D}^{2} \overline{\mathrm{D}}_{\dot{\alpha}} \Lambda_{\beta \alpha}+\frac{1}{3} \overline{\mathrm{D}}_{\dot{\alpha}} \mathrm{D}^{2} \Lambda_{\beta \alpha}
$$

and the superspace action of the linearized $4 D, \mathcal{N}=1$ non-minimal supergravity supermultiplet in this first order formulation is

$$
\begin{aligned}
& S[\mathcal{W}, \Omega, \mathcal{I}]=\int d^{8} z\left\{\mathcal{W}^{\beta \alpha \dot{\alpha}} \Omega_{\beta \alpha \dot{\alpha}}\right.+\mathcal{W}^{\beta \alpha \dot{\alpha}} \mathcal{I}_{\beta \alpha \dot{\alpha}} \\
&-c \Omega^{\beta \alpha \dot{\alpha}}\left[\overline{\mathrm{D}}^{2} \mathcal{I}_{\beta \dot{\alpha}}-\frac{4}{9} \mathrm{D}_{\beta} \overline{\mathrm{D}}^{\dot{\beta}} \overline{\mathcal{I}}_{\alpha \dot{\beta} \dot{\alpha}}-\overline{\mathrm{D}}^{\dot{\beta}} \mathrm{D}_{\beta} \overline{\mathcal{I}}_{\alpha \dot{\beta} \dot{\alpha}}\right. \\
&\left.\left.\quad+\frac{2}{9} \mathrm{D}_{\beta} \overline{\mathrm{D}}_{\dot{\alpha}} \overline{\mathcal{I}}_{\alpha}+\frac{2}{9} C_{\beta \alpha} \mathrm{D}^{\gamma} \overline{\mathrm{D}}_{\dot{\alpha}} \overline{\mathcal{I}}_{\gamma}\right]\right\}+ \text { c.c. }
\end{aligned}
$$

One can do one more step and integrate out the auxiliary connection-like superfields. The equations of motion for $\mathcal{W}_{\beta \alpha \dot{\alpha}}$ and $\Omega_{\beta \alpha \dot{\alpha}}$ are:

$$
\begin{aligned}
& \Omega_{\beta \alpha \dot{\alpha}}+\mathcal{I}_{\beta \alpha \dot{\alpha}}=0 \\
& -\frac{1}{c} \mathcal{W}_{\beta \alpha \dot{\alpha}}+\overline{\mathrm{D}}^{2} \mathcal{I}_{\beta \dot{\alpha}}-\frac{4}{9} \mathrm{D}_{\beta} \overline{\mathrm{D}}^{\dot{\beta}} \overline{\mathcal{I}}_{\alpha \dot{\beta} \dot{\alpha}}-\overline{\mathrm{D}}^{\dot{\beta}} \mathrm{D}_{\beta} \overline{\mathcal{I}}_{\alpha \dot{\beta} \dot{\alpha}}+\frac{2}{9} \mathrm{D}_{\beta} \overline{\mathrm{D}}_{\dot{\alpha}} \overline{\mathcal{I}}_{\alpha}+\frac{2}{9} C_{\beta \alpha} \mathrm{D}^{\gamma} \overline{\mathrm{D}}_{\dot{\alpha}} \overline{\mathcal{I}}_{\gamma}=0 .
\end{aligned}
$$

Substituting them in (2.43) we find the following action

$$
\begin{aligned}
S_{(\mathrm{Y}=3 / 2)}\left[\mathcal{I}_{\beta \alpha \dot{\alpha}}\right]=c \int d^{8} z\{ & \mathcal{I}^{\beta \alpha \dot{\alpha}} \overline{\mathrm{D}}^{2} \mathcal{I}_{\beta \alpha \dot{\alpha}}+\frac{2}{9} \mathcal{I}^{\beta \alpha \dot{\alpha}} \mathrm{D}_{\beta} \overline{\mathrm{D}}_{\dot{\alpha}} \overline{\mathcal{I}}_{\alpha}+c . c . \\
& \left.-\frac{8}{9} \mathcal{I}^{\beta \alpha \dot{\alpha}} \mathrm{D}_{\beta} \overline{\mathrm{D}}^{\dot{\beta}} \overline{\mathcal{I}}_{\alpha \dot{\beta} \dot{\alpha}}-2 \mathcal{I}^{\beta \alpha \dot{\alpha}} \overline{\mathrm{D}}^{\dot{\beta}} \mathrm{D}_{\beta} \overline{\mathcal{I}}_{\alpha \dot{\beta} \dot{\alpha}}+\frac{4}{9} \mathcal{I}^{\dot{\alpha}} \mathrm{D}^{\alpha} \overline{\mathrm{D}}_{\dot{\alpha}} \overline{\mathcal{I}}_{\alpha}\right\} .
\end{aligned}
$$

It is straightforward to show that this action is equal to $S_{(\mathrm{Y}=3 / 2)}\left[H_{\alpha \dot{\alpha}}, \chi_{\alpha}\right]$ with the identification $H_{\alpha \dot{\alpha}}=\mathcal{H}_{\alpha \dot{\alpha}}+\overline{\mathcal{H}}_{\alpha \dot{\alpha}}$ and $\chi_{\alpha}=\mathcal{X}_{\alpha}$. The equations of motion for $\mathcal{H}_{\alpha \dot{\alpha}}$ and $\mathcal{X}_{\alpha}$ as derived from the above action are

$$
\begin{aligned}
& \mathcal{E}_{\alpha \dot{\alpha}}^{(\mathcal{H})}=\mathrm{D}^{\beta} \overline{\mathrm{D}}^{2} \mathcal{I}_{\beta \alpha \dot{\alpha}}+\overline{\mathrm{D}}^{\dot{\beta}} \mathrm{D}^{2} \overline{\mathcal{I}}_{\alpha \dot{\beta} \dot{\alpha}}, \\
& \overline{\mathcal{E}}_{\dot{\alpha}}^{(\mathcal{X})}=\overline{\mathrm{D}}^{2} \mathcal{I}_{\dot{\alpha}}+\frac{1}{3} \mathrm{D}^{\alpha} \overline{\mathrm{D}}_{\dot{\alpha}} \overline{\mathcal{I}}_{\alpha}+\frac{2}{3} \mathrm{D}^{\alpha} \overline{\mathrm{D}}^{\dot{\beta}} \overline{\mathcal{I}}_{\alpha \dot{\beta} \dot{\alpha}}+\overline{\mathrm{D}}^{\dot{\beta}} \mathrm{D}^{\alpha} \overline{\mathcal{I}}_{\alpha \dot{\beta} \dot{\alpha}} .
\end{aligned}
$$

These equations of motion satisfy the following Bianchi identities

$$
\begin{array}{ll}
\mathrm{D}^{\alpha} \mathcal{E}_{\alpha \dot{\alpha}}^{(\mathcal{H})}=\mathrm{D}^{2} \overline{\mathcal{E}}_{\dot{\alpha}}^{(\mathcal{X})} & {[L-\text { invariance }]} \\
\mathcal{E}_{\alpha \dot{\alpha}}^{(\mathcal{H})}=\overline{\mathcal{E}}_{\alpha \dot{\alpha}}^{(\mathcal{H})} & {[\eta \text { - invariance }]} \\
\overline{\mathrm{D}}_{(\dot{\beta}} \overline{\mathcal{E}}_{\dot{\alpha})}^{(\mathcal{X})}=0 & {[\Lambda \text { - invariance }]}
\end{array}
$$

A practical advantage of action (2.45) is that it packages both $\mathcal{H}_{\alpha \dot{\alpha}}$ and $\mathcal{X}_{\alpha}$ superfields into one object $\mathcal{I}_{\beta \alpha \dot{\alpha}}$. This is a very attractive feature if we want to construct interactions. 
It is useful to compare the structures that appear in the first order formulation as described above, with the corresponding structures in the conventional superspace description of supergravity. In both cases we have unconstrained prepotential superfields, connections and an enhanced set of symmetries. However, in the first order description some of these features are motivated for different reasons.

In conventional supergravity formulation, one starts with the algebra of covariant derivatives constructed out of supervielbeins and superconnections. Then various constraints are imposed on supertorsion in order to minimize the number of independent superfields. These constraints are solved by a set of unconstrained (prepotential) superfields, which determine the superconnection and supervielbein. Moreover, the prepotentials carry additional symmetries that preserve the constraints. Besides the general super-coordinates transformation and the local super-Lorentz rotations an additional symmetry appears $(\Lambda$ symmetry). The algebraic terms of these symmetries can be used to remove unnecessary prepotentials, for example the real part of the complex vector prepotential.

From the viewpoint of the linearized theory (2.20), the same prepotentials emerge as the appropriate variables for the Lagrangian description of the corresponding irreducible representation, as determined by the diagonalization of the Casimir operators of supersymmetry algebra. In this configuration there is no longer an algebraic local symmetry and the rest of the surviving symmetries are linearized and take the form of gauge transformations (2.21).

In the first order formulation (2.43) the notion of an algebraic local symmetry is restored by complexifying the vector prepotential and introduce the local $\eta$-transformation (2.24) in order to preserve the degrees of freedom of the theory. In this case, the complexification is motivated by the 'first order' philosophy which probes for invariant quantities with less derivatives than the equations of motion. Moreover, the connection-like superfields are introduced at the level of the action as auxiliary superfields that help us achieve $\eta$-invariance and are determined by their algebraic equations of motion. Interestingly, this formulation can be extended to higher spin supermultiplets, in contrast to the covariant derivative approach of supergravity in which this extension is not obvious. We will not explore this direction in this work, but it will be investigated in a follow up paper.

\section{Trivial symmetries and Noether's procedure}

\subsection{Review of trivial symmetries with examples in supersymmetric theories}

Let us consider a theory that includes multiple copies of the same kind of fields. Then, we can streamline the notation and combine all these fields into one object $\phi^{I}$ by introducing an index $I=1,2, \ldots$ that counts them. For simplicity we suppress all other types of indices that these fields may carry. ${ }^{7}$ Now let us consider the following transformation

$$
\delta_{\lambda} \phi^{I}=\lambda C^{I J} \frac{\delta S}{\delta \phi^{J}}
$$

\footnotetext{
${ }^{7}$ Index $I$ as introduced here may appear to be an internal index. However all arguments can be restated even if $I$ is replaced by spinorial or spacetime indices. They also count the number of components of the fields.
} 
where $\lambda$ is the parameter that controls the transformation, $C^{I J}$ is an appropriate collection of coefficients and $\frac{\delta S}{\delta \phi^{J}}$ is the equation of motion for field $\phi^{J}$. The variation of the action of this theory $S\left[\phi^{I}\right]$ under the above $\lambda$-transformation (with appropriate boundary conditions) is

$$
\delta_{\lambda} S=\int \lambda C^{I J} \frac{\delta S}{\delta \phi^{J}} \frac{\delta S}{\delta \phi^{I}}
$$

By choosing appropriately the symmetry property of $C^{I J}$ this variation vanishes. For example, if $\phi^{I}$ are commuting [anticommuting] fields then by choosing $C^{I J}$ to be antisymmetric $\left(C^{I J}=-C^{J I}\right)\left[\right.$ symmetric $\left.\left(C^{I J}=C^{J I}\right)\right]$ we get $\delta_{\lambda} S=0$. Hence the $\lambda$-transformation is a symmetry of the theory.

These transformations are called trivial symmetries and form an infinite class of obvious invariants that every theory has. Trivial symmetries do not reduce the number of physical degrees of freedom, for this reason one is usually not interested in such transformations and they are not even included in the set of symmetries. However, it can be fruitful to be aware of these trivial symmetries when constructing interactions. In some cases, the parameter $\lambda$ can be proportional to the coupling constant of the interaction and hence the combination of the trivial symmetries with Noether's procedure can reveal properties about the structure of the interaction vertex. A successful application of this approach can be found in [73].

Furthermore, because the commutator of infinitesimal symmetries is also an infinitesimal symmetry of the action, trivial symmetries may appear in the right hand side of the commutators of generators of symmetries. Famously, this is the case in some formulations of supersymmetric theories where one can find deformations of the usual supersymmetry algebra by terms proportional to the equations of motion. This phenomenon is sometimes called as 'on-shell SUSY closure'. In all such cases, these deformation terms are precisely trivial symmetries that emerge in the right hand side of the commutator. The identifying characteristic is not only that they are proportional to the equations of motion but also crucially the proportionality coefficients have the correct symmetry property to make them trivial symmetries. ${ }^{8}$ In fact, from the view point of supersymmetric theories formulated in a non manifestly manner (such as the component formulation) one way to justify the existence of auxiliary fields is the removal of these trivial symmetry deformations of the algebra such that we get honest representations of supersymmetry algebra.

A less known application of trivial symmetries in supersymmetric theories has to do with a special class of theories with auxiliary fermions in their off-shell spectrum. Nonminimal supergravity is such a theory, as well as its higher spin cousins. As mentioned previously, the auxiliary fermions come in pairs $(\beta, \rho)$ with different engineering dimensions and the action includes a term $\beta^{\alpha} \rho_{\alpha}$. In that case, there is a trivial symmetry between the auxiliary fermions $\beta, \rho$ and the dynamical fermions $\psi$ with the transformation parameter being dimensionless. ${ }^{9}$ For these theories, the trivial symmetries can be used to simplify

\footnotetext{
${ }^{8}$ For example the commutator of two supersymmetry transformations of the chiral multiplet fermion after integrating out the auxiliary fields takes the form $\left\{\epsilon^{a} \mathcal{Q}_{a}, \eta^{b} \mathcal{Q}_{b}\right\} \psi^{c} \sim i\left(\epsilon \gamma^{m} \eta\right) \partial_{m} \psi^{c}+\underbrace{\left(\epsilon \gamma^{n} \eta\right)}_{\lambda} \underbrace{\left(\gamma_{n}\right)^{c d}}_{C^{c d}=C^{d c}} \frac{\delta S}{\delta \psi^{d}}$.

${ }^{9}$ The simplest example is the complex linear supermultiplet. The transformation $\delta \beta=c \gamma^{m} \partial_{m} \psi$, $\delta \psi=-c \rho, \delta \rho=0$ is a trivial symmetry of the theory. Notice that the equation of motion of the auxiliary fermion $\beta$ is $\mathcal{E}^{(\beta)}=\rho$.
} 
the supersymmetry transformation of the dynamical fermion $\delta_{Q} \psi$ (by removing all terms proportional to $\delta_{Q} \rho$ ).

\subsection{Application to electromagnetic interactions}

A characteristic feature of electromagnetic interactions of various fields is that because of the underlying $\mathrm{U}(1)$ (locally isomorphic to $\mathrm{SO}(2)$ ) symmetry, these fields come in doublets. Therefore trivial symmetries like (3.1) emerge. In this case, we have a doublet of $Y=3 / 2$ supermultiplets labeled by an internal index $i$ which takes two values. Hence, following the discussion above there are trivial symmetries that preserve the sum of the two free actions. An example of such a trivial symmetry is

$$
\delta H_{\alpha \dot{\alpha}}^{i}=\frac{\lambda}{M^{2}} \epsilon^{i j} \mathcal{E}_{\alpha \dot{\alpha}}^{j(H)}
$$

where $\mathcal{E}_{\alpha \dot{\alpha}}^{j(H)}$ is the equation of motion of $H_{\alpha \dot{\alpha}}^{j},{ }^{10} M$ is an appropriate mass scale required by engineering dimensions, $\lambda$ is a dimensionless, arbitrary, real superfield and $\epsilon^{i j}$ is the unique (up to an overall factor) two dimensional, rank 2, antisymmetric tensor. Although we know that this transformation is nothing more than a trivial symmetry, because it introduces a dimensionful parameter and is linear in superfield $H_{\alpha \dot{\alpha}}$ it can be interpreted as a trivial, ${ }^{11}$ first order (in the coupling constant) correction to the free gauge transformations. In this case, the coupling constant is $1 / M^{2}$ which we know from Metsaev's classification [1] is the correct coupling constant for the cubic interaction between two spin 2 gauge fields and a spin 1 gauge field involving three spacetime derivatives. This is also consistent with the non-supersymmetric 2-2-1 vertex [72, 73] which will be included in the supersymmetric $3 / 2-3 / 2-1 / 2$ vertex that we are constructing.

In this interpretation of (3.3), the dimensionless real scalar superfield $\lambda$ is a function of the free gauge transformation parameters. For the vector multiplet the gauge parameter is an arbitrary dimensionless chiral superfield $\Phi\left(\delta V=\Phi+\bar{\Phi}, \Phi=\overline{\mathrm{D}}^{2} L\right)$ hence we can write

$$
\delta_{1}^{(\Phi)} H_{\alpha \dot{\alpha}}^{i}=\frac{c}{M^{2}}(\Phi+\bar{\Phi}) \epsilon^{i j} \mathcal{E}_{j \alpha \dot{\alpha}}^{(H)} .
$$

Using Noether's procedure of constructing consistent interactions perturbatively we must satisfy the following gauge consistency condition for cubic vertices

$$
\delta_{0}^{(\xi)} S_{1}+\delta_{1}^{(\xi)} S_{0}=0
$$

where $\delta_{0}^{(\xi)}, \delta_{1}^{(\xi)}$ are the zeroth and first order variations with respect to transformations controlled by parameter $\xi$. $S_{0}, S_{1}$ are the free and first order correction actions respectively. Applying this for $\xi=\Phi$ and using the fact that (3.4) is a trivial symmetry $\left(\delta_{1}^{(\Phi)} S_{0}=0\right)$ we conclude that the cubic interaction must be trivially invariant under the free gauge transformation of the vector multiplet

$$
\delta_{0}^{(\Phi)} S_{1}=0
$$

The only way this can be true is if the interaction vertex depends on the gauge invariant superfield strength $W_{\alpha}=\overline{\mathrm{D}}^{2} \mathrm{D}_{\alpha} V$ instead of the bare vector superfield $V$.

\footnotetext{
${ }^{10}$ We can also write this trivial symmetry in terms of the complexified superfield $\mathcal{H}_{\alpha \dot{\alpha}}$ introduced in 2.3 .

${ }^{11}$ Trivial because it does not correspond to a non-trivial interaction vertex.
} 


\section{Electromagnetic interaction of non-minimal linearized supergravity}

\subsection{Preparations}

Our aim is to find a consistent cubic interaction between the vector multiplet and a doublet of linearized non-minimal supergravity multiplets. For the description of the vector multiplet and the two supergravity multiplets we will use the formulations developed in 2.2 and 2.3. Our starting action is the sum of free actions (2.45) and (2.17)

$$
S_{0}=\sum_{i=1}^{2} S_{(\mathrm{Y}=3 / 2)}\left[\mathcal{I}_{\beta \alpha \dot{\alpha}}^{i}\right]+c S_{(\mathrm{Y}=1 / 2)}\left[\mathcal{I}_{\alpha}\right]
$$

where $c$ is a fixed relative coefficient between the two free actions which ensures that (up to an overall sign) both actions have positive kinetic energy. We search for $S_{1}$, the first order correction to $S_{0}$ in the coupling constant expansion. We know that it will be quadratic in the supergravity superfields, linear in the vector multiplet, the coupling constant is $1 / M^{2}$ and it depends on the superfield strength $W_{\alpha}=\overline{\mathrm{D}}^{2} \mathrm{D}_{\alpha} V\left(S_{1}=S_{1}\left[\mathcal{I}_{\beta \alpha \dot{\alpha}}^{i}, W_{\alpha}\right]\right)$. Also, a quick dimensional analysis argument shows that it must involve 3 supersymmetric covariant derivatives distributed among $\mathcal{I}_{\beta \alpha \dot{\alpha}}^{i}$ and $W_{\alpha}$. Finally, it must satisfy the consistency conditions

$$
\delta_{1}^{(\eta)} S_{0}+\delta_{0}^{(\eta)} S_{1}=0, \delta_{1}^{(\Lambda)} S_{0}+\delta_{0}^{(\Lambda)} S_{1}=0
$$

where $\delta_{0}^{(\eta)}, \delta_{0}^{(\Lambda)}$ are the variations according to transformations (2.27) and (2.37) respectively and $\delta_{1}^{(\eta)}, \delta_{1}^{(\Lambda)}$ are appropriate first order corrections to the corresponding free transformations. Equations (4.2) must be solved simultaneously for $S_{1}, \delta_{1}^{(\eta)}, \delta_{1}^{(\Lambda)}$.

A conventional approach to solve these equations is to make an ansatz for $S_{1}$ and calculate it's variation under free gauge transformations with the assumption that the onshell equations of motion for the free theory hold. On-shell, Noether's constraints simplify to $\left.\delta_{0} S_{1}\right|_{\delta S_{0}=0}=0$ which can be solved by fixing the coefficients in our ansatz. Having found a non-trivial $S_{1}$ then the calculation can be repeated off-shell in order to find $\delta_{1}$. In our case, just writing a non-trivial ansatz with all possible index contractions and various derivative distributions generates a very large number of terms that can not be controlled easily. For this reason, we will start in the opposite end and consider various ansatz for the first order corrections of the transformation laws. There are not many terms that one can write for $\delta_{1}$ and even if we do not include everything, just a few terms are enough to get us started and generate everything else in the process. This procedure will give us clues regarding $S_{1}$.

For the corrections to the $\eta$ transformation of superfield $\mathcal{H}_{\alpha \dot{\alpha}}^{i}$ we can write

$$
\delta_{1}^{(\eta)} \mathcal{H}_{\alpha \dot{\alpha}}^{i}=i \frac{d}{M^{2}} \epsilon^{i j} \eta_{j \dot{\alpha}}^{\beta} \mathrm{D}_{(\beta} W_{\alpha)}+\ldots
$$

where the dots correspond to additional terms that we can write. Let us consider the effect of this deformation in the transformation law by varying only $\mathcal{H}_{\alpha \dot{\alpha}}^{i}$ in $S_{0}$. After some 
lengthy calculations we find

$$
\begin{aligned}
\delta_{1}^{(\eta)} S_{0}=-\frac{i d}{M^{2}} \int d^{8} z \epsilon^{i j}\left\{\mathrm{D}^{\beta} \eta_{(\beta}^{j \dot{\alpha}} W^{\alpha} \mathcal{E}_{\alpha) \dot{\alpha}}^{i(\mathcal{H})}-\eta_{(\beta}^{j} \dot{\alpha} \mathrm{D}^{\alpha} \overline{\mathrm{D}}^{\dot{\gamma}} \mathrm{D}^{\beta} \overline{\mathcal{I}}_{\alpha) \dot{\gamma} \dot{\alpha}}^{i} \mathcal{E}^{(\mathcal{V})}\right. \\
\left.+\mathrm{D}_{\rho} \eta_{(\beta}^{j} \dot{\alpha} W^{\rho}\left[\mathrm{D}^{\alpha} \overline{\mathrm{D}}^{\dot{\gamma}} \mathrm{D}^{\beta} \overline{\mathcal{I}}_{\alpha) \dot{\gamma} \dot{\alpha}}^{i}-\mathrm{D}^{\alpha} \overline{\mathrm{D}}^{2} \mathcal{I}^{i \beta}{ }_{\alpha) \dot{\alpha}}\right]\right\}+c . c .
\end{aligned}
$$

The first two terms are proportional to the equations of motion of superfields $\mathcal{H}_{\alpha \dot{\alpha}}^{i}$ and $\mathcal{V}$, hence they can be compensated by adding appropriate corrections in their corresponding transformation laws. The rest of the terms are non-trivial because they can only be canceled by $\delta_{0}^{(\eta)} S_{1}$. This is a suggestion to consider the following ansatz for $S_{1}$

$$
S_{1}^{(a)}=\frac{1}{M^{2}} \int d^{8} z \epsilon^{i j} \mathcal{I}_{\rho(\beta}^{j}{ }^{\dot{\alpha}} W^{\rho}\left(\mathrm{D}^{\alpha} \overline{\mathrm{D}}^{\dot{\gamma}} \mathrm{D}^{\beta} \overline{\mathcal{I}}_{\alpha) \dot{\gamma} \dot{\alpha}}^{i}-\mathrm{D}^{\alpha} \overline{\mathrm{D}}^{2} \mathcal{I}_{\alpha) \dot{\alpha}}^{i \beta}\right)+c . c .
$$

Using (2.27), one can show that the zeroth order variation of the terms inside the parenthesis vanishes, hence $\delta_{0}^{(\eta)} S_{1}^{(a)}$ matches precisely the terms in the second line of (4.4). Therefore an appropriate choice of parameter $d$ will solve the $\eta$-Noether consistency condition in (4.2).

For the $\Lambda$-Noether condition, we calculate $\delta_{0}^{\Lambda} S_{1}^{(a)}$. Again after some algebra we find

$$
\begin{aligned}
\delta_{0}^{(\Lambda)} S_{1}^{(a)}=\frac{1}{M^{2}} \int d^{8} z \epsilon^{i j}\left\{\overline{\mathrm{D}}_{\dot{\rho}} \bar{\Lambda}^{j \dot{\rho} \dot{\alpha}} W^{\beta} \mathcal{E}_{\beta \dot{\alpha}}^{i(\mathcal{H})}+2 \mathrm{D}_{\beta}\left(\overline{\mathrm{D}}_{\dot{\rho}} \bar{\Lambda}^{j \dot{\rho} \dot{\alpha}} W^{\beta}\right) \overline{\mathcal{E}}_{\dot{\alpha}}^{i(\mathcal{X})}\right. \\
-\frac{1}{3} \overline{\mathrm{D}}_{\dot{\rho}} \bar{\Lambda}^{j \dot{\rho} \dot{\alpha}} W^{\alpha}\left[2 \mathrm{D}^{2} \overline{\mathrm{D}}_{\dot{\alpha}} \overline{\mathcal{I}}_{\alpha}^{i}+\mathrm{D}^{2} \overline{\mathrm{D}}^{\dot{\gamma}} \overline{\mathcal{I}}_{\alpha \dot{\gamma} \dot{\alpha}}^{i}\right] \\
\left.+\mathcal{I}^{j \rho \beta \dot{\alpha}} W_{\rho} \mathrm{D}^{2} \overline{\mathrm{D}}_{\dot{\alpha}} \mathrm{D}^{\alpha} \Lambda_{\alpha \beta}^{i}\right\}+ \text { c.c. }
\end{aligned}
$$

Observe that both terms in the fist line are proportional to the equation of motion of $\mathcal{H}_{\alpha \dot{\alpha}}^{i}$ and $\mathcal{X}_{\alpha}^{i}$ superfields, hence these terms can be eliminated by appropriate deformations in there $\Lambda$-transformations. However, the rest of the terms are not of this kind, hence $S_{1}^{(a)}$ is not enough to solve (4.2), we have to consider additional contributions. Equation (4.6) provides a hint for such an additional contribution

$$
S_{1}^{(b)}=\frac{g}{M^{2}} \int d^{8} z \epsilon^{i j} \mathcal{I}_{\rho}^{j}{ }^{\gamma \dot{\alpha}} \mathcal{W}^{\rho} \mathrm{D}^{2}\left(2 \overline{\mathrm{D}}_{\dot{\alpha}} \overline{\mathcal{I}}_{\gamma}^{i}+\overline{\mathrm{D}}^{\dot{\gamma}} \overline{\mathcal{I}}_{\gamma \dot{\gamma} \dot{\alpha}}^{i}\right)+\text { c.c. }
$$

It is straightforward to check that under transformations $(2.37), \delta_{0}^{(\Lambda)} S_{1}^{(b)}$ matches precisely the last two lines of (4.6) up to an overall constant which can be adjusted appropriately and thus solving the $\Lambda$-Noether constraint.

However, by adding $S_{1}^{(b)}$ we have ruined the $\eta$-invariance, thus one must repeat the above calculations for the updated $S_{1}$ ansatz

$$
S_{1}=S_{1}^{(a)}+S_{1}^{(b)} .
$$

In general, we may have to keep adding terms $S_{1}^{(c)}, S_{1}^{(d)}, \ldots$ in order to counteract the effect of previous term. However in this case, terms $S_{1}^{(a)}$ and $S_{1}^{(b)}$ are enough to satisfy (4.2) and to determine the deformations of the gauge transformations. 


\subsection{Cubic interaction $3 / 2-3 / 2-1 / 2$}

Consider the ansatz

$$
\begin{array}{r}
S_{1}=\frac{1}{M^{2}} \int d^{8} z \epsilon^{i j}\left\{\mathcal{I}_{\rho(\beta}^{j} \dot{\alpha} W^{\rho} \mathrm{D}^{\alpha}\left(\overline{\mathrm{D}}^{\dot{\gamma}} \mathrm{D}^{\beta} \overline{\mathcal{I}}_{\alpha) \dot{\gamma} \dot{\alpha}}^{i}-\overline{\mathrm{D}}^{2} \mathcal{I}^{i \beta}{ }_{\alpha) \dot{\alpha}}\right)\right. \\
\left.+g \mathcal{I}_{\rho}^{j}{ }^{\gamma \dot{\alpha}} \mathcal{W}^{\rho} \mathrm{D}^{2}\left(2 \overline{\mathrm{D}}_{\dot{\alpha}} \overline{\mathcal{I}}_{\gamma}^{i}+\overline{\mathrm{D}}^{\dot{\gamma}} \overline{\mathcal{I}}_{\gamma \dot{\gamma} \dot{\alpha}}^{i}\right)\right\}+c . c .
\end{array}
$$

where an overall constant can be absorbed in the definition of the coupling constant. We calculate it's $\Lambda$ and $\eta$-variations using (2.37) and (2.27) respectively:

$$
\begin{aligned}
\delta_{0}^{(\Lambda)} S_{1}=\frac{1}{M^{2}} \int d^{8} z \epsilon^{i j}\{ & 2 \mathrm{D}_{\rho}\left(\overline{\mathrm{D}}_{\dot{\sigma}} \overline{\Lambda^{j \sigma \alpha}} W^{\rho}\right) \overline{\mathcal{E}}_{\dot{\alpha}}^{i(\mathcal{X})} \\
& +\overline{\mathrm{D}}_{\dot{\sigma}} \bar{\Lambda}^{j \dot{\sigma} \dot{\alpha}} W^{\rho} \mathcal{E}_{\rho \dot{\alpha}}^{i(\mathcal{H})} \\
& +(3 g-1) \mathcal{I}_{\rho}^{j \beta \dot{\alpha}} W^{\rho} \mathrm{D}^{\alpha} \overline{\mathrm{D}}_{\dot{\alpha}} \mathrm{D}^{2} \Lambda_{\beta \alpha}^{i} \\
& \left.-\left(g-\frac{1}{3}\right) \overline{\mathrm{D}}_{\dot{\sigma}} \bar{\Lambda}^{j \sigma \dot{\alpha}} W^{\rho} \mathrm{D}_{\rho}\left(2 \mathrm{D}^{\alpha} \overline{\mathrm{D}}_{\dot{\alpha}} \overline{\mathcal{I}}_{\alpha}^{i}+\mathrm{D}^{\alpha} \overline{\mathrm{D}}^{\dot{\gamma}} \overline{\mathcal{I}}_{\alpha \dot{\gamma} \dot{\alpha}}^{i}\right)\right\}+ \text { c.c. } \\
\delta_{0}^{(\eta)} S_{1}=\frac{i}{M^{2}} \int d^{8} z \epsilon^{i j}\{[ & \eta_{(\beta}^{j} \dot{\alpha}\left(\mathrm{D}^{a} \overline{\mathrm{D}}^{\dot{\gamma}} \mathrm{D}^{\beta} \overline{\mathcal{I}}_{\alpha) \dot{\gamma} \dot{\alpha}}^{i}-\mathrm{D}^{\alpha} \overline{\mathrm{D}}^{2} \mathcal{I}^{i \beta}{ }_{\alpha) \dot{\alpha}}\right) \\
& \left.+g \eta^{j \gamma \dot{\alpha}} \mathrm{D}^{2}\left(2 \overline{\mathrm{D}}_{\dot{\alpha}} \overline{\mathcal{I}}_{\gamma}^{i}+\overline{\mathrm{D}}^{\dot{\gamma}} \overline{\mathcal{I}}_{\gamma \dot{\gamma} \dot{\alpha}}^{i}\right)\right] \mathcal{E}^{(\mathcal{V})} \\
& \left.-\mathrm{D}^{\beta}\left(\eta_{(\beta}^{j \dot{\alpha}} W^{\alpha}\right) \mathcal{E}_{\alpha) \dot{\alpha}}^{i(\mathcal{H})}\right\}+c . c .
\end{aligned}
$$

Similarly, the variation of $S_{0}$ under the first order transformations

$$
\delta_{1} S_{0}=\int d^{8} z\left\{-2 \delta_{1} \mathcal{H}^{i \alpha \dot{\alpha}} \mathcal{E}_{\alpha \dot{\alpha}}^{i(\mathcal{H})}+2 \delta_{1} \overline{\mathcal{X}}^{i \dot{\alpha}} \overline{\mathcal{E}}_{\dot{\alpha}}^{i(\mathcal{X})}-2 c \delta_{1} \mathcal{V} \mathcal{E}^{(\mathcal{V})}\right\}+\text { c.c. }
$$

Therefore, (4.2) are solved by choosing $g=\frac{1}{3}$ and

$$
\begin{aligned}
& \delta_{1}^{(\eta)} \mathcal{H}^{i \alpha \dot{\alpha}}=-\frac{i}{2 M^{2}} \epsilon^{i j} \mathrm{D}^{(\beta}\left(\eta_{\beta}^{j \dot{\alpha}} W^{\alpha)}\right), \quad \delta_{1}^{(\Lambda)} \mathcal{H}^{i \alpha \dot{\alpha}}=\frac{1}{2 M^{2}} \epsilon^{i j} \overline{\mathrm{D}}_{\dot{\sigma}} \bar{\Lambda}^{j \dot{\sigma} \dot{\alpha}} W^{\alpha}, \\
& \delta_{1}^{(\eta)} \overline{\mathcal{X}}^{i \dot{\alpha}}=0, \quad \delta_{1}^{(\Lambda)} \overline{\mathcal{X}}^{i \dot{\alpha}}=-\frac{\epsilon^{i j}}{M^{2}} \mathrm{D}_{\rho}\left(\overline{\mathrm{D}}_{\dot{\sigma}} \bar{\Lambda}^{j \dot{\sigma} \dot{\alpha}} W^{\rho}\right), \\
& \delta_{1}^{(\eta)} \mathcal{V}=\frac{i \epsilon^{i j}}{2 c M^{2}}\left[\eta_{(\beta}^{j}{ }^{\dot{\alpha}} \mathrm{D}^{\alpha}\left(\overline{\mathrm{D}}^{\dot{\gamma}} \mathrm{D}^{\beta} \overline{\mathcal{I}}_{\alpha) \dot{\gamma} \dot{\alpha}}^{i}-\overline{\mathrm{D}}^{2} \mathcal{I}^{i \beta}{ }_{\alpha) \dot{\alpha}}\right)+\frac{1}{3} \eta^{j \gamma \dot{\alpha}} \mathrm{D}^{2}\left(2 \overline{\mathrm{D}}_{\dot{\alpha}} \overline{\mathcal{I}}_{\gamma}^{i}+\overline{\mathrm{D}}^{\dot{\gamma}} \overline{\mathcal{I}}_{\gamma \dot{\gamma} \dot{\alpha}}^{i}\right)\right], \delta_{1}^{(\Lambda)} \mathcal{V}=0
\end{aligned}
$$

Observe that $\delta_{1}^{(\eta)} \mathcal{H}_{\alpha \dot{\alpha}}^{i}$ has a symmetrization between $\beta$ and $\alpha$ indices. If we expand the symmetrization and write explicitly the two terms we see that one of them $\left(\mathrm{D}_{\alpha}\left[\frac{i}{2 M^{2}} \epsilon^{i j} \eta_{\dot{\alpha}}^{j \beta} W_{\beta}\right]\right)$ is just a free gauge transformation (2.23) for some specific value of the gauge parameter $\bar{L}_{\dot{\alpha}} \sim \frac{i}{M^{2}} \epsilon^{i j} \eta^{j \beta}{ }_{\alpha} W_{\beta}$. Such terms of course correspond to just a redefinition of the gauge parameter and we will not include them in the set of non-trivial corrections to the gauge transformations. 
Moreover, notice that $\delta_{1}^{(\Lambda)} \overline{\mathcal{X}}_{\dot{\alpha}}^{i}$ can be expanded by distributing the covariant derivative giving two terms. One of these terms is $\frac{1}{M^{2}} \epsilon^{i j} \overline{\mathrm{D}}^{\dot{\sigma}} \bar{\Lambda}_{\dot{\sigma} \dot{\alpha}}^{j} \mathcal{E}^{(\mathcal{V})}$ where $\mathcal{E}^{(\mathcal{V})}=\mathrm{D}^{\rho} W_{\rho}$. Of course, this is nothing more than a trivial symmetry that our theory has. It is easy to check that the following transformations preserve $S_{0}$ :

$$
\delta \overline{\mathcal{X}}_{\dot{\alpha}}^{i}=\frac{d}{M^{2}} \epsilon^{i j} \overline{\mathrm{D}}^{\dot{\sigma}} \bar{\Lambda}_{\dot{\sigma} \dot{\alpha}}^{j} \mathcal{E}^{(\mathcal{V})}, \delta \mathcal{V}=\frac{d}{c M^{2}} \epsilon^{i j} \overline{\mathrm{D}}^{\dot{\sigma}} \bar{\Lambda}_{\dot{\sigma}}^{j \dot{\alpha}} \overline{\mathcal{E}}_{\dot{\alpha}}^{j(\mathcal{X})}
$$

Therefore, this part of the transformation does not correspond to non-trivial deformations of the free gauge transformations and will also be ignored.

To conclude, the cubic interaction between a doublet of linearized non-minimal supergravity multiplets $(Y=3 / 2)$ and the vector multiplet $(Y=1 / 2)$ has the form

$$
\begin{array}{r}
S_{1}=\frac{1}{M^{2}} \int d^{8} z \epsilon^{i j}\left\{\mathcal{I}_{\rho(\beta}^{j}{ }^{\dot{\alpha}} W^{\rho} \mathrm{D}^{\alpha}\left(\overline{\mathrm{D}}^{\dot{\gamma}} \mathrm{D}^{\beta} \overline{\mathcal{I}}_{\alpha) \dot{\gamma} \dot{\alpha}}^{i}-\overline{\mathrm{D}}^{2} \mathcal{I}^{i \beta}{ }_{\alpha) \dot{\alpha}}\right)\right. \\
\left.+\frac{1}{3} \mathcal{I}_{\rho}^{j}{ }^{\gamma \dot{\alpha}} W^{\rho} \mathrm{D}^{2}\left(2 \overline{\mathrm{D}}_{\dot{\alpha}} \overline{\mathcal{I}}_{\gamma}^{i}+\overline{\mathrm{D}}^{\dot{\gamma}} \overline{\mathcal{I}}_{\gamma \dot{\gamma} \dot{\alpha}}^{i}\right)\right\}+c . c .
\end{array}
$$

This interaction is by construction manifestly invariant under transformations (2.6) and (2.23), however invariance under the $\eta$ and $\Lambda$-transformations require the following deformations:

$$
\begin{aligned}
& \delta_{1} \mathcal{H}_{\alpha \dot{\alpha}}^{i}=-\frac{i}{M^{2}} \epsilon^{i j} \mathrm{D}^{\beta}\left(\eta_{\beta \dot{\alpha}}^{j} W_{\alpha}\right)-\frac{1}{2 M^{2}} \epsilon^{i j} \overline{\mathrm{D}}^{\dot{\sigma}} \bar{\Lambda}_{\dot{\alpha} \dot{\alpha}}^{j} W_{\alpha}, \\
& \delta_{1} \overline{\mathcal{X}}_{\dot{\alpha}}^{i}=-\frac{1}{M^{2}} \epsilon^{i j} \mathrm{D}^{\rho} \overline{\mathrm{D}}^{\dot{\sigma}} \bar{\Lambda}_{\dot{\sigma} \dot{\alpha}}^{j} W_{\rho}, \\
& \delta_{1} \mathcal{V}=\frac{i \epsilon^{i j}}{2 c M^{2}}\left[\eta_{(\beta}^{j}{ }^{\dot{\alpha}} \mathrm{D}^{\alpha}\left(\overline{\mathrm{D}}^{\dot{\gamma}} \mathrm{D}^{\beta} \overline{\mathcal{I}}_{\alpha) \dot{\gamma} \dot{\alpha}}^{i}-\overline{\mathrm{D}}^{2} \mathcal{I}^{i \beta}{ }_{\alpha) \dot{\alpha}}\right)+\frac{1}{3} \eta^{j \gamma \dot{\alpha}} \mathrm{D}^{2}\left(2 \overline{\mathrm{D}}_{\dot{\alpha}} \overline{\mathcal{I}}_{\gamma}^{i}+\overline{\mathrm{D}}^{\dot{\gamma}} \overline{\mathcal{I}}_{\gamma \dot{\gamma} \dot{\alpha}}^{i}\right)\right] .
\end{aligned}
$$

\section{Conclusions}

Motivated by the success of the frame-like formulation in constructing consistent and nontrivial interactions between various higher spin gauge fields, we develop a superspace first order formulation for the linearized supergravity and vector supermultiplets. This is done by relaxing the reality property of the corresponding superfields in order to simplify the gauge transformation laws ( $L$-transformation) and help us define simple invariants that are used to write manifestly invariant actions. Of course, by doing that we introduce new, unwanted, degrees of freedom which we eliminate by introducing a new local symmetry ( $\eta$-transformation). Making the action invariant under this local symmetry leads to the introduction of connection-like auxiliary superfields. In this case due to their fermionic nature we have a pair of such 'connections' $\mathcal{W}_{\beta \alpha \dot{\alpha}}$ and $\Omega_{\beta \alpha \dot{\alpha}}$. After integrating them out, we find a Lagrangian description for the $Y=3 / 2$ multiplet in terms of a single superfield $\mathcal{I}_{\beta \alpha \dot{\alpha}}$ which packages together the complexified prepotential and its compensator 


$$
\begin{aligned}
\mathcal{I}_{\beta \alpha \dot{\alpha}}=\mathrm{D}_{\beta} \mathcal{H}_{\alpha \dot{\alpha}}+C_{\beta \alpha} \overline{\mathcal{X}}_{\dot{\alpha}}\left(\text { and } \mathcal{I}_{\dot{\alpha}}=C^{\beta \alpha} \mathcal{I}_{\beta \alpha \dot{\alpha}}\right) \\
S_{(\mathrm{Y}=3 / 2)}\left[\mathcal{I}_{\beta \alpha \dot{\alpha}}\right]=c \int d^{8} z\left\{\mathcal{I}^{\beta \alpha \dot{\alpha}} \overline{\mathrm{D}}^{2} \mathcal{I}_{\beta \alpha \dot{\alpha}}+\frac{2}{9} \mathcal{I}^{\beta \alpha \dot{\alpha}} \mathrm{D}_{\beta} \overline{\mathrm{D}}_{\dot{\alpha}} \overline{\mathcal{I}}_{\alpha}+c . c .\right. \\
\left.\quad-\frac{8}{9} \mathcal{I}^{\beta \alpha \dot{\alpha}} \mathrm{D}_{\beta} \overline{\mathrm{D}}^{\dot{\beta}} \overline{\mathcal{I}}_{\alpha \dot{\beta} \dot{\alpha}}-2 \mathcal{I}^{\beta \alpha \dot{\alpha}} \overline{\mathrm{D}}^{\dot{\beta}} \mathrm{D}_{\beta} \overline{\mathcal{I}}_{\alpha \dot{\beta} \dot{\alpha}}+\frac{4}{9} \mathcal{I}^{\dot{\alpha}} \mathrm{D}^{\alpha} \overline{\mathrm{D}}_{\dot{\alpha}} \overline{\mathcal{I}}_{\alpha}\right\} .
\end{aligned}
$$

By construction this action is manifestly invariant under the $L$-transformation and also invariant under the $\eta$-transformation. Moreover, $\mathcal{I}_{\beta \alpha \dot{\alpha}}$ satisfies two Bianchi identities which give rise to an additional emerging symmetry ( $\Lambda$-transformations) which matches what is expected from the prepotential description viewpoint.

We emphasize that the frame formulation in superspace has a long history. The full, non-linear theory of supergravity was developed in superspace using supervielbein and superconnection superfields. These superfields carry many additional degrees of freedom and one is forced to impose several (conventional) constraints on them. After solving these constraints in terms of prepotentials and linearizing the theory, all features of the first order formulation have been dissolved. Our approach brings back some of these notions and not only allows us to construct cubic interactions but also creates a path to bring the power of first order formulation to various higher spin supermultiplets. This task will be completed in a following paper.

In this paper we apply this alternative description to construct cubic electromagnetic interaction of supergravity. As always, fields that interact electromagnetically come in doublets and for this reason various trivial symmetries emerge. These are symmetries that every field theory which involves many fields has, but because they do not reduce the number of degrees of freedom they are not discussed often. In this case, trivial symmetries can be used to show that the $3 / 2-3 / 2-1 / 2$ cubic vertex between two non-minimal superspin $Y=3 / 2$ multiplets (linearized non-minimal supergravity) and one superspin $Y=1 / 2$ multiplet (vector multiplet) must depend on the gauge invariant superfield strength of the vector multiplet $W_{\alpha}$. This feature generalizes previously known results for the nonsupersymmetric cubic vertex interactions of two spin 2 particles with a spin 1 particle.

Using Noether's procedure we find the first order correction to the action which describes the above cubic interaction to be

$$
\begin{aligned}
S_{1}=\frac{1}{M^{2}} \int d^{8} z \epsilon^{i j}\left\{\mathcal{I}_{\rho(\beta}^{j}{ }^{\dot{\alpha}}\right. & W^{\rho} \mathrm{D}^{\alpha}\left(\overline{\mathrm{D}}^{\dot{\gamma}} \mathrm{D}^{\beta} \overline{\mathcal{I}}_{\alpha) \dot{\gamma} \dot{\alpha}}^{i}-\overline{\mathrm{D}}^{2} \mathcal{I}^{i \beta}{ }_{\alpha) \dot{\alpha}}\right) \\
+ & \left.\frac{1}{3} \mathcal{I}_{\rho}^{j}{ }^{\gamma \dot{\alpha}} W^{\rho} \mathrm{D}^{2}\left(2 \overline{\mathrm{D}}_{\dot{\alpha}} \overline{\mathcal{I}}_{\gamma}^{i}+\overline{\mathrm{D}}^{\dot{\gamma}} \overline{\mathcal{I}}_{\gamma \dot{\gamma} \dot{\alpha}}^{i}\right)\right\}+c . c .
\end{aligned}
$$

This cubic interaction requires non-trivial deformations of the $\eta$ and $\Lambda$ gauge transformations as follows:

$$
\begin{aligned}
& \delta_{1} \mathcal{H}_{\alpha \dot{\alpha}}^{i}=-\frac{i}{M^{2}} \epsilon^{i j} \mathrm{D}^{\beta}\left(\eta_{\beta \dot{\alpha}}^{j} W_{\alpha}\right)-\frac{1}{2 M^{2}} \epsilon^{i j} \overline{\mathrm{D}}^{\dot{\sigma}} \bar{\Lambda}_{\dot{\sigma} \dot{\alpha}}^{j} W_{\alpha}, \\
& \delta_{1} \overline{\mathcal{X}}_{\dot{\alpha}}^{i}=-\frac{1}{M^{2}} \epsilon^{i j} \mathrm{D}^{\rho} \overline{\mathrm{D}}^{\dot{\sigma}} \bar{\Lambda}_{\dot{\sigma} \dot{\alpha}}^{j} W_{\rho}, \\
& \delta_{1} \mathcal{V}=\frac{i \epsilon^{i j}}{2 c M^{2}}\left[\eta_{(\beta}^{j}{ }^{\dot{\alpha}} \mathrm{D}^{\alpha}\left(\overline{\mathrm{D}}^{\dot{\gamma}} \mathrm{D}^{\beta} \overline{\mathcal{I}}_{\alpha) \dot{\gamma} \dot{\alpha}}^{i}-\overline{\mathrm{D}}^{2} \mathcal{I}^{i \beta}{ }_{\alpha) \dot{\alpha}}\right)+\frac{1}{3} \eta^{j \gamma \dot{\alpha}} \mathrm{D}^{2}\left(2 \overline{\mathrm{D}}_{\dot{\alpha}} \overline{\mathcal{I}}_{\gamma}^{i}+\overline{\mathrm{D}}^{\dot{\gamma}} \overline{\mathcal{I}}_{\gamma \dot{\gamma} \dot{\alpha}}^{i}\right)\right] .
\end{aligned}
$$


These deformations can be mapped to the conventional superfield description according to the rule

$$
H_{\alpha \dot{\alpha}}^{i}=\mathcal{H}_{\alpha \dot{\alpha}}^{i}+\overline{\mathcal{H}}_{\alpha \dot{\alpha}}^{i}, \chi_{\alpha}^{i}=\mathcal{X}_{\alpha}^{i}, V=\mathcal{V}+\overline{\mathcal{V}}, \eta_{\alpha \dot{\alpha}}^{i}=\frac{i}{2}\left(\mathrm{D}_{\alpha} \bar{L}_{\dot{\alpha}}^{i}+\overline{\mathrm{D}}_{\dot{\alpha}} L_{\alpha}^{i}\right)
$$

This interaction is of the type $\mathcal{L}_{1} \sim \Phi_{1} \Phi_{2} W_{3}$ as discussed in the introduction and can be used as the starting point for higher spin generalizations of it. These interactions are known to exist and have been constructed for non-supersymmetric theories and theories with onshell supersymmetry. This work hopefully provides the right set of tools needed in order to construct these interactions in superspace with manifest supersymmetry. Furthermore, it would be interesting to investigate whether the first order description of supergravity given in 2.3 can be pushed beyond the linearized limit and make contact with the full non-linear theory. Also its applications to minimal formulations of supergravity and higher spin multiplets should be studied. It is known that one can exchange formulations of supergravity - go from non-minimal supergravity $(n=-1)$ to old-minimal supergravity $(n=-1 / 3)$ and vice versa - by exploiting the duality between chiral and complex linear superfields. An example of employing this duality can be found in [64] where consistent interactions between matter and higher spin supermultiplets are constructed. Using similar arguments one may find how to extend the above results to a minimal formulation of supergravity.

\section{Acknowledgments}

The work of I. L. B was partially supported by Ministry of Education of Russian Federation, project FEWF-2020-0003. The work of K. K. and S. J. G. is supported in part by the endowment of the Ford Foundation Professorship of Physics at Brown University. Also K. K. gratefully acknowledges the support of the Brown Theoretical Physics Center.

Open Access. This article is distributed under the terms of the Creative Commons Attribution License (CC-BY 4.0), which permits any use, distribution and reproduction in any medium, provided the original author(s) and source are credited.

\section{References}

[1] R.R. Metsaev, Cubic interaction vertices of massive and massless higher spin fields, Nucl. Phys. B 759 (2006) 147 [hep-th/0512342] [INSPIRE].

[2] R.R. Metsaev, Cubic interaction vertices for fermionic and bosonic arbitrary spin fields, Nucl. Phys. B 859 (2012) 13 [arXiv:0712.3526] [INSPIRE].

[3] R.R. Metsaev, Light-cone gauge cubic interaction vertices for massless fields in AdS $S_{4}$, Nucl. Phys. B 936 (2018) 320 [arXiv:1807.07542] [InSPIRE].

[4] K. Mkrtchyan, Cubic interactions of massless bosonic fields in three dimensions, Phys. Rev. Lett. 120 (2018) 221601 [arXiv: 1712.10003] [INSPIRE].

[5] P. Kessel and K. Mkrtchyan, Cubic interactions of massless bosonic fields in three dimensions II: Parity-odd and Chern-Simons vertices, Phys. Rev. D 97 (2018) 106021 [arXiv: 1803.02737] [INSPIRE].

[6] R.R. Metsaev, Cubic interaction vertices for $N=1$ arbitrary spin massless supermultiplets in flat space, JHEP 08 (2019) 130 [arXiv:1905.11357] [INSPIRE]. 
[7] R.R. Metsaev, Cubic interactions for arbitrary spin $\mathcal{N}$-extended massless supermultiplets in 4d flat space, JHEP 11 (2019) 084 [arXiv:1909.05241] [INSPIRE].

[8] F.A. Berends, G.J.H. Burgers and H. van Dam, Explicit Construction of Conserved Currents for Massless Fields of Arbitrary Spin, Nucl. Phys. B 271 (1986) 429 [InSPIRE].

[9] G. Barnich and M. Henneaux, Consistent couplings between fields with a gauge freedom and deformations of the master equation, Phys. Lett. B 311 (1993) 123 [hep-th/9304057] [INSPIRE].

[10] X. Bekaert, E. Joung and J. Mourad, On higher spin interactions with matter, JHEP 05 (2009) 126 [arXiv:0903.3338] [InSPIRE].

[11] X. Bekaert and E. Meunier, Higher spin interactions with scalar matter on constant curvature spacetimes: conserved current and cubic coupling generating functions, JHEP 11 (2010) 116 [arXiv:1007.4384] [INSPIRE].

[12] I.L. Buchbinder, T.V. Snegirev and Y.M. Zinoviev, Cubic interaction vertex of higher-spin fields with external electromagnetic field, Nucl. Phys. B 864 (2012) 694 [arXiv:1204.2341] [INSPIRE].

[13] E. Joung, L. Lopez and M. Taronna, Solving the Noether procedure for cubic interactions of higher spins in (A)dS, J. Phys. A 46 (2013) 214020 [arXiv:1207.5520] [InSPIRE].

[14] E. Joung and M. Taronna, Cubic-interaction-induced deformations of higher-spin symmetries, JHEP 03 (2014) 103 [arXiv:1311.0242] [INSPIRE].

[15] I.L. Buchbinder, A. Fotopoulos, A.C. Petkou and M. Tsulaia, Constructing the cubic interaction vertex of higher spin gauge fields, Phys. Rev. D 74 (2006) 105018 [hep-th/0609082] [inSPIRE].

[16] X. Bekaert, N. Boulanger, S. Cnockaert and S. Leclercq, On killing tensors and cubic vertices in higher-spin gauge theories, Fortsch. Phys. 54 (2006) 282 [hep-th/0602092] [INSPIRE].

[17] A. Fotopoulos, N. Irges, A.C. Petkou and M. Tsulaia, Higher-Spin Gauge Fields Interacting with Scalars: The Lagrangian Cubic Vertex, JHEP 10 (2007) 021 [arXiv:0708.1399] [INSPIRE].

[18] A. Fotopoulos and M. Tsulaia, Gauge Invariant Lagrangians for Free and Interacting Higher Spin Fields. A review of the BRST formulation, Int. J. Mod. Phys. A 24 (2009) 1 [arXiv:0805.1346] [INSPIRE].

[19] D. Polyakov, Interactions of Massless Higher Spin Fields From String Theory, Phys. Rev. D 82 (2010) 066005 [arXiv:0910.5338] [INSPIRE].

[20] M. Henneaux, G. Lucena Gómez and R. Rahman, Higher-Spin Fermionic Gauge Fields and Their Electromagnetic Coupling, JHEP 08 (2012) 093 [arXiv:1206.1048] [INSPIRE].

[21] R.R. Metsaev, BRST-BV approach to cubic interaction vertices for massive and massless higher-spin fields, Phys. Lett. B 720 (2013) 237 [arXiv:1205.3131] [INSPIRE].

[22] M. Henneaux, G. Lucena Gómez and R. Rahman, Gravitational Interactions of Higher-Spin Fermions, JHEP 01 (2014) 087 [arXiv:1310.5152] [INSPIRE].

[23] I.L. Buchbinder, V.A. Krykhtin, M. Tsulaia and D. Weissman, Cubic Vertices for $N=1$ Supersymmetric Massless Higher Spin Fields in Various Dimensions, Nucl. Phys. B 967 (2021) 115427 [arXiv:2103.08231] [INSPIRE].

[24] I.L. Buchbinder and A.A. Reshetnyak, General cubic interacting vertex for massless integer higher spin fields, Phys. Lett. B 820 (2021) 136470 [arXiv:2105.12030] [InSPIRE]. 
[25] M.A. Vasiliev, Gauge form of description of massless fields with arbitrary spin (in Russian), Sov. J. Nucl. Phys. 32 (1980) 439 [inSPIRE].

[26] E.S. Fradkin and M.A. Vasiliev, On the Gravitational Interaction of Massless Higher Spin Fields, Phys. Lett. B 189 (1987) 89 [InSPIRE].

[27] V.E. Lopatin and M.A. Vasiliev, Free Massless Bosonic Fields of Arbitrary Spin in d-dimensional de Sitter Space, Mod. Phys. Lett. A 3 (1988) 257 [InSPIRE].

[28] M.A. Vasiliev, Free Massless Fermionic Fields of Arbitrary Spin in d-dimensional de Sitter Space, Nucl. Phys. B 301 (1988) 26 [InSPIRE].

[29] M.A. Vasiliev, Cubic interactions of bosonic higher spin gauge fields in $A d S_{5}$, Nucl. Phys. B 616 (2001) 106 [Erratum ibid. 652 (2003) 407] [hep-th/0106200] [INSPIRE].

[30] K.B. Alkalaev and M.A. Vasiliev, $N=1$ supersymmetric theory of higher spin gauge fields in $A d S_{5}$ at the cubic level, Nucl. Phys. B 655 (2003) 57 [hep-th/0206068] [INSPIRE].

[31] Y.M. Zinoviev, Frame-like gauge invariant formulation for massive high spin particles, Nucl. Phys. B 808 (2009) 185 [arXiv: 0808.1778] [INSPIRE].

[32] D.S. Ponomarev and M.A. Vasiliev, Frame-Like Action and Unfolded Formulation for Massive Higher-Spin Fields, Nucl. Phys. B 839 (2010) 466 [arXiv:1001.0062] [INSPIRE].

[33] Y.M. Zinoviev, Spin 3 cubic vertices in a frame-like formalism, JHEP 08 (2010) 084 [arXiv:1007.0158] [INSPIRE].

[34] N. Boulanger, E.D. Skvortsov and Y.M. Zinoviev, Gravitational cubic interactions for a simple mixed-symmetry gauge field in AdS and flat backgrounds, J. Phys. A 44 (2011) 415403 [arXiv:1107.1872] [INSPIRE].

[35] Y.M. Zinoviev, Gravitational cubic interactions for a massive mixed symmetry gauge field, Class. Quant. Grav. 29 (2012) 015013 [arXiv:1107.3222] [INSPIRE].

[36] I.L. Buchbinder, M.V. Khabarov, T.V. Snegirev and Y.M. Zinoviev, Lagrangian formulation of the massive higher spin $N=1$ supermultiplets in AdS $S_{4}$ space, Nucl. Phys. B 942 (2019) 1 [arXiv: 1901.09637] [INSPIRE].

[37] I.L. Buchbinder, M.V. Khabarov, T.V. Snegirev and Y.M. Zinoviev, Lagrangian description of the partially massless higher spin $N=1$ supermultiplets in $A d S_{4}$ space, JHEP 08 (2019) 116 [arXiv: 1904.01959] [INSPIRE].

[38] I.L. Buchbinder, M.V. Khabarov, T.V. Snegirev and Y.M. Zinoviev, Lagrangian formulation for the infinite spin $N=1$ supermultiplets in $d=4$, Nucl. Phys. B 946 (2019) 114717 [arXiv: 1904.05580] [INSPIRE].

[39] I.L. Buchbinder and T.V. Snegirev, Lagrangian formulation of free arbitrary $N$-extended massless higher spin supermultiplets in 4D, AdS space, Symmetry 12 (2020) 2052 [arXiv : 2009.00896] [INSPIRE].

[40] M.V. Khabarov and Y.M. Zinoviev, Massless higher spin cubic vertices in flat four dimensional space, JHEP 08 (2020) 112 [arXiv:2005.09851] [INSPIRE].

[41] M.V. Khabarov and Y.M. Zinoviev, On massive spin-2 in the Fradkin-Vasiliev formalism. II. General massive case, arXiv:2107.05900 [INSPIRE].

[42] P.A.M. Dirac, Relativistic wave equations, Proc. Roy. Soc. Lond. A 155 (1936) 447.

[43] M. Fierz and W. Pauli, On relativistic wave equations for particles of arbitrary spin in an electromagnetic field, Proc. Roy. Soc. Lond. A 173 (1939) 211. 
[44] E.P. Wigner, On Unitary Representations of the Inhomogeneous Lorentz Group, Annals Math. 40 (1939) 149 [INSPIRE].

[45] V. Bargmann and E.P. Wigner, Group Theoretical Discussion of Relativistic Wave Equations, Proc. Nat. Acad. Sci. 34 (1948) 211 [INSPIRE].

[46] L.P.S. Singh and C.R. Hagen, Lagrangian formulation for arbitrary spin. 1. The boson case, Phys. Rev. D 9 (1974) 898 [InSPIRE].

[47] L.P.S. Singh and C.R. Hagen, Lagrangian formulation for arbitrary spin. 2. The fermion case, Phys. Rev. D 9 (1974) 910 [inSPIRE].

[48] C. Fronsdal, Massless Fields with Integer Spin, Phys. Rev. D 18 (1978) 3624 [InSPIRE].

[49] J. Fang and C. Fronsdal, Massless Fields with Half Integral Spin, Phys. Rev. D 18 (1978) 3630 [INSPIRE].

[50] B. de Wit and D.Z. Freedman, Systematics of Higher Spin Gauge Fields, Phys. Rev. D 21 (1980) 358 [INSPIRE].

[51] M.A. Vasiliev, Free Massless Fields of Arbitrary Spin in the de Sitter Space and Initial Data for a Higher Spin Superalgebra, Fortsch. Phys. 35 (1987) 741 [INSPIRE].

[52] S.M. Kuzenko and A.G. Sibiryakov, Massless gauge superfields of higher integer superspins, JETP Lett. 57 (1993) 539 [INSPIRE].

[53] S.M. Kuzenko, A.G. Sibiryakov and V.V. Postnikov, Massless gauge superfields of higher half integer superspins, JETP Lett. 57 (1993) 534 [INSPIRE].

[54] S.M. Kuzenko and A.G. Sibiryakov, Free massless higher superspin superfields on the anti-de Sitter superspace, Phys. Atom. Nucl. 57 (1994) 1257 [arXiv:1112.4612] [InSPIRE].

[55] S.J. Gates Jr., S.M. Kuzenko and A.G. Sibiryakov, $N=2$ supersymmetry of higher superspin massless theories, Phys. Lett. B 412 (1997) 59 [hep-th/9609141] [INSPIRE].

[56] S.J. Gates Jr. and K. Koutrolikos, On $4 D, \mathcal{N}=1$ massless gauge superfields of arbitrary superhelicity, JHEP 06 (2014) 098 [arXiv:1310.7385] [INSPIRE].

[57] S.J. Gates Jr. and K. Koutrolikos, On 4D, $N=1$ Massless Gauge Superfields of Higher Superspin: Half-Odd-Integer Case, arXiv:1310.7386 [INSPIRE].

[58] K. Koutrolikos, Aspects of Supersymmetric Higher Spins, PoS CORFU2014 (2015) 152 [INSPIRE].

[59] K. Koutrolikos, Superspace formulation of massive half-integer superspin, JHEP 03 (2021) 254 [arXiv: 2012.12225] [INSPIRE].

[60] I.L. Buchbinder, S.J. Gates Jr. and K. Koutrolikos, Hierarchy of Supersymmetric Higher Spin Connections, Phys. Rev. D 102 (2020) 125018 [arXiv: 2010. 02061] [inSPIRE].

[61] I.L. Buchbinder, S.J. Gates Jr. and K. Koutrolikos, Higher Spin Superfield interactions with the Chiral Supermultiplet: Conserved Supercurrents and Cubic Vertices, Universe 4 (2018) 6 [arXiv: 1708.06262] [INSPIRE].

[62] J. Hutomo and S.M. Kuzenko, Non-conformal higher spin supercurrents, Phys. Lett. B 778 (2018) 242 [arXiv: 1710.10837] [INSPIRE].

[63] J. Hutomo and S.M. Kuzenko, The massless integer superspin multiplets revisited, JHEP 02 (2018) 137 [arXiv: 1711.11364] [InSPIRE].

[64] K. Koutrolikos, P. Kočí and R. von Unge, Higher Spin Superfield interactions with Complex linear Supermultiplet: Conserved Supercurrents and Cubic Vertices, JHEP 03 (2018) 119 [arXiv: 1712.05150] [INSPIRE]. 
[65] I.L. Buchbinder, S.J. Gates Jr. and K. Koutrolikos, Interaction of supersymmetric nonlinear sigma models with external higher spin superfields via higher spin supercurrents, JHEP 05 (2018) 204 [arXiv: 1804.08539] [INSPIRE].

[66] E.I. Buchbinder, J. Hutomo and S.M. Kuzenko, Higher spin supercurrents in anti-de Sitter space, JHEP 09 (2018) 027 [arXiv: 1805.08055] [INSPIRE].

[67] I.L. Buchbinder, S.J. Gates Jr. and K. Koutrolikos, Integer superspin supercurrents of matter supermultiplets, JHEP 05 (2019) 031 [arXiv:1811.12858] [INSPIRE].

[68] J. Hutomo, S.M. Kuzenko and D. Ogburn, $\mathcal{N}=2$ supersymmetric higher spin gauge theories and current multiplets in three dimensions, Phys. Rev. D 98 (2018) 125004 [arXiv: 1807.09098] [INSPIRE].

[69] I.L. Buchbinder, S.J. Gates Jr. and K. Koutrolikos, Conserved higher spin supercurrents for arbitrary spin massless supermultiplets and higher spin superfield cubic interactions, JHEP 08 (2018) 055 [arXiv: 1805.04413] [INSPIRE].

[70] S.J. Gates Jr. and K. Koutrolikos, Progress on cubic interactions of arbitrary superspin supermultiplets via gauge invariant supercurrents, Phys. Lett. B 797 (2019) 134868 [arXiv: 1904.13336] [INSPIRE].

[71] M.V. Khabarov and Y.M. Zinoviev, Cubic interaction vertices for massless higher spin supermultiplets in $d=4$, JHEP 02 (2021) 167 [arXiv: 2012.00482] [INSPIRE].

[72] N. Boulanger, S. Leclercq and P. Sundell, On The Uniqueness of Minimal Coupling in Higher-Spin Gauge Theory, JHEP 08 (2008) 056 [arXiv: 0805.2764] [INSPIRE].

[73] Y.M. Zinoviev, On spin 2 electromagnetic interactions, Mod. Phys. Lett. A 24 (2009) 17 [arXiv:0806.4030] [INSPIRE].

[74] Y.M. Zinoviev, On massive spin 2 electromagnetic interactions, Nucl. Phys. B 821 (2009) 431 [arXiv: 0901.3462] [INSPIRE].

[75] Y.M. Zinoviev, On electromagnetic interactions for massive mixed symmetry field, JHEP 03 (2011) 082 [arXiv: 1012.2706] [INSPIRE].

[76] J. Wess and B. Zumino, Superspace Formulation of Supergravity, Phys. Lett. B 66 (1977) 361 [INSPIRE].

[77] W. Siegel and S.J. Gates Jr., Superfield Supergravity, Nucl. Phys. B 147 (1979) 77 [inSPIRE].

[78] V.I. Ogievetsky and E.S. Sokatchev, The Simplest Group of Einstein Supergravity, Sov. J. Nucl. Phys. 31 (1980) 140 [INSPIRE].

[79] S.J. Gates Jr., M.T. Grisaru, M. Roček and W. Siegel, Superspace Or One Thousand and One Lessons in Supersymmetry, vol. 58 of Frontiers in Physics (1983) [hep-th/0108200] [INSPIRE].

[80] I.L. Buchbinder and S.M. Kuzenko, Ideas and methods of supersymmetry and supergravity: A Walk through superspace, CRC Press (1995).

[81] M. Rausch de Traubenberg and M. Valenzuela, A Supergravity Primer: From Geometrical Principles to the Final Lagrangian, WSP, Singapur (2020), [DOI] [INSPIRE].

[82] S.J. Gates Jr. and K. Koutrolikos, From Diophantus to Supergravity and massless higher spin multiplets, JHEP 11 (2017) 063 [arXiv:1707.00194] [INSPIRE].

[83] S.J. Gates Jr. and K. Koutrolikos, A dynamical theory for linearized massive superspin 3/2, JHEP 03 (2014) 030 [arXiv: 1310.7387] [INSPIRE]. 